\title{
Radiative properties of a plasma moving across a magnetic field. II: Numerical results
}

\author{
Robert Roussel-Dupré \\ Space Science and Technology Division, Los Alamos National Laboratory, Los Alamos, \\ New Mexico 87545 \\ Ronald H. Miller \\ Space Physics Research Laboratory, Department of Atmospheric, Oceanic and Space Sciences, \\ The University of Michigan, Ann Arbor, Michigan 48109-2143
}

(Received 23 April 1992; accepted 22 December 1992)

\begin{abstract}
A theoretical analysis developed in a companion paper to treat the early-time evolution of plasmas moving across a background magnetic field is applied to the modeling of lowbeta, barium chemical releases in the magnetosphere. The results indicate that radiation damping plays an important role in defining the plasma cloud evolution, causing a rapid decay of the polarization field and a loss of plasma kinetic energy and momentum on time scales comparable to several ion gyroperiods. The radiation spectrum consists of a burst of chirped, high-frequency (in the range of the cloud plasma frequencies) waves, followed by a pulse of whistler waves, and subsequently by ion cyclotron emission.

Scaling laws are derived for the plasma momentum and energy loss rates and predictions for the braking time, the amplitude and spectrum of the radiation field, and the total radiated power are presented for conditions relevant to the recent Combined Release and Radiation Effects Satellite (CRRES) experiments [Phys. Fluids B 4, 2249 (1992)].
\end{abstract}

\section{INTRODUCTION}

In a companion paper ${ }^{1}$ (hereafter, Paper I), we presented the details of a theoretical analysis that was specifically developed to address the early-time ( $<100$ ion gyroperiods) evolution of plasmas moving across a background magnetic field, including both the dynamics associated with the formation of a polarization field and the generation and propagation of electromagnetic waves. Previous analyses of this problem have either ignored the radiation field altogether or simply assumed without justification that the polarization field reaches a steady-state value within an ion gyroperiod with a magnitude that permits the plasma cloud to propagate freely across the magnetic field. Given the analysis and results presented in $\mathrm{Pa}$ per $I$, it is our intention in this paper to demonstrate that, in the presence of radiation damping, the polarization field does not achieve a steady-state value and that the radiation field can carry away a substantial amount of the plasma cloud's momentum and kinetic energy on time scales of the order of the ion gyroperiod, depending on the plasma dimensions. It is interesting to note that high-frequency (lower-hybrid) electromagnetic radiation has been measured emanating from barium releases in the magnetosphere, ${ }^{2,3}$ from barium releases in the ionosphere, ${ }^{3-6}$ and from "magnetic holes" formed in the magnetopause. ${ }^{7}$ The precise source of this radiation, however, is not well understood. A rapid decay of the polarization field has also been measured in some of these experiments. ${ }^{3}$

Several approximations are made in our description of the plasma dynamics and our treatment of the propagation of electromagnetic waves. First, the plasma is assumed to have a Maxwellian velocity distribution function and only the moments of the distribution function (density, mean velocity, and temperature) are evolved in time (by means of the fluid equations). As a result we do not include the effects of kinetic instabilities or resonant wave-particle interactions. Second, the plasma dimensions are assumed to be much larger than an ion gyroradius and we therefore ignore the additional current systems and corresponding electromagnetic radiation that arise in connection with momentum and energy transport processes driven by density, velocity, and temperature gradients. Third, the production rate of additional plasma and plasma momentum resulting, for example, from photoionization of neutrals that are moving across the magnetic field is assumed to be small compared to an ion gyrofrequency. This effect can therefore be neglected over time scales small compared to an ion gyroperiod. However, in treating the self-consistent evolution of the plasma and the radiation field over longer time scales it is important to include it. Fourth, the initial dimensions of the plasma are assumed to evolve in time in a way that is consistent with the neglect of pressure and velocity gradients. In general, this effect can be omitted over short time scales, however, plasma dimensions are observed to change over longer time scales (e.g., in active experiments) and, as in the case of plasma production, this effect must be included in order to treat the self-consistent evolution of the plasma and the radiation field over the longer times of interest. Fifth, the plasma is assumed to be collisionless. Sixth, the effects of a background plasma are omitted. Depending on its density relative to that of the plasma cloud, the background plasma can both neutralize the polarization field and affect the propagation of electromagnetic radiation launched by the cloud. Seventh, the dimensions of the plasma cloud are assumed to be large compared to the vacuum wavelength or dielectric wave- 
length of radiation launched during the early phases of evolution of the plasma. This approximation allows us to treat the propagation of electromagnetic radiation in the limit of geometrical optics. In addition, because the primary source of radiation is the oscillating polarization field perpendicular to the magnetic field, the electromagnetic waves are assumed to propagate primarily along the magnetic field. This fact allows for an expansion of the fields in terms of Hermite polynomials, which as the order of the expansion increases, incorporates additional effects associated with smaller and smaller scale structures in the direction of the polarization field. With these approximations, the results obtained in Paper I are only applicable to a limited set of experiments and natural phenomena. Nevertheless, our present results do contain the essential physics needed to assess the importance of radiation dynamics in the overall evolution of cross-field plasma motion and, in addition, provide an excellent nucleus around which a more comprehensive analysis can be developed. In particular, it is our intention in future studies to add the effects of a background plasma, extend the expansion in terms of Hermite polynomials to higher order, allow for a slow evolution of the plasma dimensions resulting from expansion of the plasma clouds, and incorporate a source term in the continuity equation to model plasma production.

The results obtained in Paper I are immediately applicable to the analysis of low- $\beta$ ( $=$ initial cross-field plasma energy density/background magnetic field energy density) chemical releases in the magnetosphere such as were obtained during the recent Chemical Release and Radiation Effects Satellite (CRRES) winter releases. ${ }^{8}$ We do not, however, attempt in this paper to provide a detailed comparison between theory and experiment simply because the relevant data analysis has not been performed. Instead, we will discuss theoretical results that lie within the appropriate parameter regime for magnetospheric barium releases; provide appropriate scaling laws, and identify some of the potential observables. Among the issues that will be addressed quantitatively are the amplitude and spectral content of the polarization and radiation fields, the momentum and energy stored and transported away by the fields, the braking of the plasma cloud by radiation damping, and the formation of plasma structure resulting from differential braking.

In an effort to elucidate the essential role of the radiation field in this problem, we first examine in some detail the electrostatic limit of our analysis in Sec. II and then proceed in Sec. III to discuss the additional effects introduced by the electromagnetic radiation. In the electrostatic limit, the magnitude and temporal evolution of the polarization field are readily obtained as is the partitioning of momentum and energy between the plasma and the fields. The electrostatic and electromagnetic results are discussed and compared in Sec. IV and relevant scaling laws are presented. The implications of our results for future analyses of cross-field plasma motion and for the interpretation of existing observations are addressed in Sec. V.

\section{ELECTROSTATIC LIMIT}

The electrostatic limit of our solution is found by setting induction electric and magnetic fields; namely, the perturbation magnetic field and the $y$ component of the electric field, to zero. In this limit, Maxwell's equations reduce to

$$
\begin{aligned}
& \nabla \cdot \mathbf{E}=4 \pi\left(q_{i} n_{i}+q_{e} n_{e}\right), \\
& \nabla \cdot \mathbf{B}=0, \\
& \nabla \times \mathbf{E}=\overline{0}, \\
& 0=4 \pi \mathbf{J}+\frac{\partial \mathbf{E}}{\partial t},
\end{aligned}
$$

while the constitutive relations in Laplace transform space are given by Eq. (11) of Paper I with the Hall conductivity, $\sigma_{\mathbf{H}}=0$; that is,

$$
\widetilde{\mathbf{J}}=\boldsymbol{\sigma} \cdot \widetilde{\mathbf{E}}+\frac{\sigma_{\mathrm{P}}}{s} \frac{V_{0} B_{0}}{c} \hat{x},
$$

where $\sigma$ is a tensor given by

$$
\boldsymbol{\sigma}=\left[\begin{array}{ccc}
\sigma_{\mathrm{P}} & 0 & 0 \\
0 & \sigma_{\mathrm{P}} & 0 \\
0 & 0 & \sigma_{\|}
\end{array}\right]
$$

with

$$
\begin{aligned}
& \sigma_{\mathrm{P}}=\frac{n_{e}(t=0) e^{2}}{m_{e}} \frac{s}{\omega_{c e}}\left(\frac{\omega_{c i}}{\left(s^{2}+\omega_{c i}^{2}\right)}+\frac{\omega_{c e}}{\left(s^{2}+\omega_{c e}^{2}\right)}\right), \\
& \sigma_{\|}=\frac{n_{e}(t=0) e^{2}}{m_{e}} \frac{\left(1+m_{e} / m_{i}\right)}{s},
\end{aligned}
$$

and where $s$ is the Laplace transform variable, $\omega_{c i}$ and $\omega_{c e}$ are the ion and electron gyrofrequencies, respectively, $n_{e}$ is the plasma density [taken initially to be $n_{e}(t=0)=n_{0}$ $e^{-x^{2} / D^{2}} e^{-z^{2} / d^{2}}$, where $D$ and $d$ are the $1 / e$ half-widths of the density profile in the $x$ and $z$ directions, respectively], $V_{0}$ is the initial velocity of the plasma across the magnetic field, $B_{0}$ is the magnitude of the background field, $c$ is the speed of light, $e$ is the magnitude of the electron charge, and $m_{e}$ and $m_{i}$ are the electron and ion mass, respectively. We note that the Hall conductivity is set to zero in order to ensure that the $y$ component of the electric field be zero, as required in the electrostatic limit and by the fact that the plasma is chosen to be of infinite extent in the $y$ direction.

Faraday's induction equation, Eq. (3), is not satisfied initially, simply because the polarization field set up in the $x$ direction by charge separation (driven by the cross-field plasma motion) varies in the $z$ direction. In general, it takes a finite amount of time for the charge layers to adjust and for a steady-state curl-free electric field configuration to be established over the length of the plasma. While it is not evident that such a configuration is even attainable in general, it is clear that the radiation field plays an important role in communicating information about the evolution of charge layers across the plasma as a whole. The earliest possible time for the plasma to establish a steady- 
state is given approximately by the time $\tau_{c}$ that it takes to communicate a local perturbation across the length of the cloud; namely, $\tau_{c} \approx d / c=\left(d / \rho_{i}\right)\left(V_{0} / c\right) \tau_{i}$ where $\rho_{i}$ is an ion gyroradius and $\tau_{i}$ is an ion gyroperiod. For our analysis, the plasma cloud dimension is assumed to be much larger than an ion gyroradius so that $d / \rho_{i}>100$. For many problems of interest, $V_{0} / c$ is of order $10^{-5}$ and $\tau_{c}$ is therefore of order $10^{-3} \tau_{i}$, corresponding, in general, to lowerhybrid time scales. Thus the radiation field can be an important factor in defining the overall evolution of plasma clouds out to time scales at least of this order. For the analysis in this section, we ignore the radiation field and compute the electric fields from Eq. (4).

Taking the Laplace transform of Eq. (4) and substituting the constitutive relations $[\mathrm{Eq} .(5)]$ yields,

$$
\begin{aligned}
& \widetilde{E}_{y}=0, \\
& \widetilde{E}_{z}=0, \\
& \widetilde{E}_{x}=\frac{\left(\sigma_{\mathrm{P}} / s\right)}{s-4 \pi \sigma_{P}} \frac{V_{0} B_{0}}{c} .
\end{aligned}
$$

Taking the inverse Laplace transform of Eq. (11) and assuming $m_{\mathrm{e}} / m_{i} \ll 1$, we find

$$
\frac{E_{x}(t, x, z)}{V_{0} B_{0} / c}=A\left(\cos \omega_{\mathrm{UH}} t-\cos \omega_{*} t\right)+B\left(\cos \omega_{*} t-1\right),
$$

where

$$
\begin{aligned}
& A=\omega_{p}^{2} / \omega_{\mathrm{UH}}^{2}, \\
& B=\omega_{P}^{2} /\left(\omega_{p}^{2}+\omega_{\mathrm{LH}}^{2}\right),
\end{aligned}
$$

and

$$
\begin{aligned}
& \omega_{\mathrm{UH}}^{2}=\omega_{p e}^{2}+\omega_{p i}^{2}+\omega_{c e}^{2}+\omega_{c i}^{2}, \\
& \omega_{p}^{2}=\omega_{p e}^{2}+\omega_{p i}^{2}, \\
& \omega_{\mathrm{LH}}^{2}=\omega_{c e} \omega_{c i}, \\
& \omega_{*}^{2}=\omega_{\mathrm{LH}}^{2}\left(\omega_{p}^{2}+\omega_{\mathrm{LH}}^{2}\right) / \omega_{\mathrm{UH}}^{2}
\end{aligned}
$$

To illustrate the behavior of this solution for different ratios of the parameter $r=\omega_{p e} / \omega_{c e}$, we plot the coefficients $A$ and $B$ and the frequency modes $\omega_{\mathrm{UH}}$ and $\omega_{\bullet}$ vs $r$ in Figs. 1 (a) and 1(b). At high plasma densities (large $r$ ), both coefficients are approximately equal to 1 and the normalized polarization field oscillates at the upper-hybrid frequency about a value equal to -1 with an amplitude equal to 1 . In this limit, the plasma can supply sufficient charge to the local charge layers to create an electric field that allows the plasma to $E \times B$ drift unimpeded across the background magnetic field. For extremely low densities $(r \ll 1)$, the polarization field oscillates at both the electron and ion cyclotron frequencies but is dominated by ion cyclotron oscillations with a normalized amplitude that is less than unity. In this case, the plasma cannot supply enough charge to the charge layers and does not drift as a whole but instead gyrates around an equilibrium position, exchanging momentum with the fields. An intermediate situation is achieved for values of $r$ between these two ex-
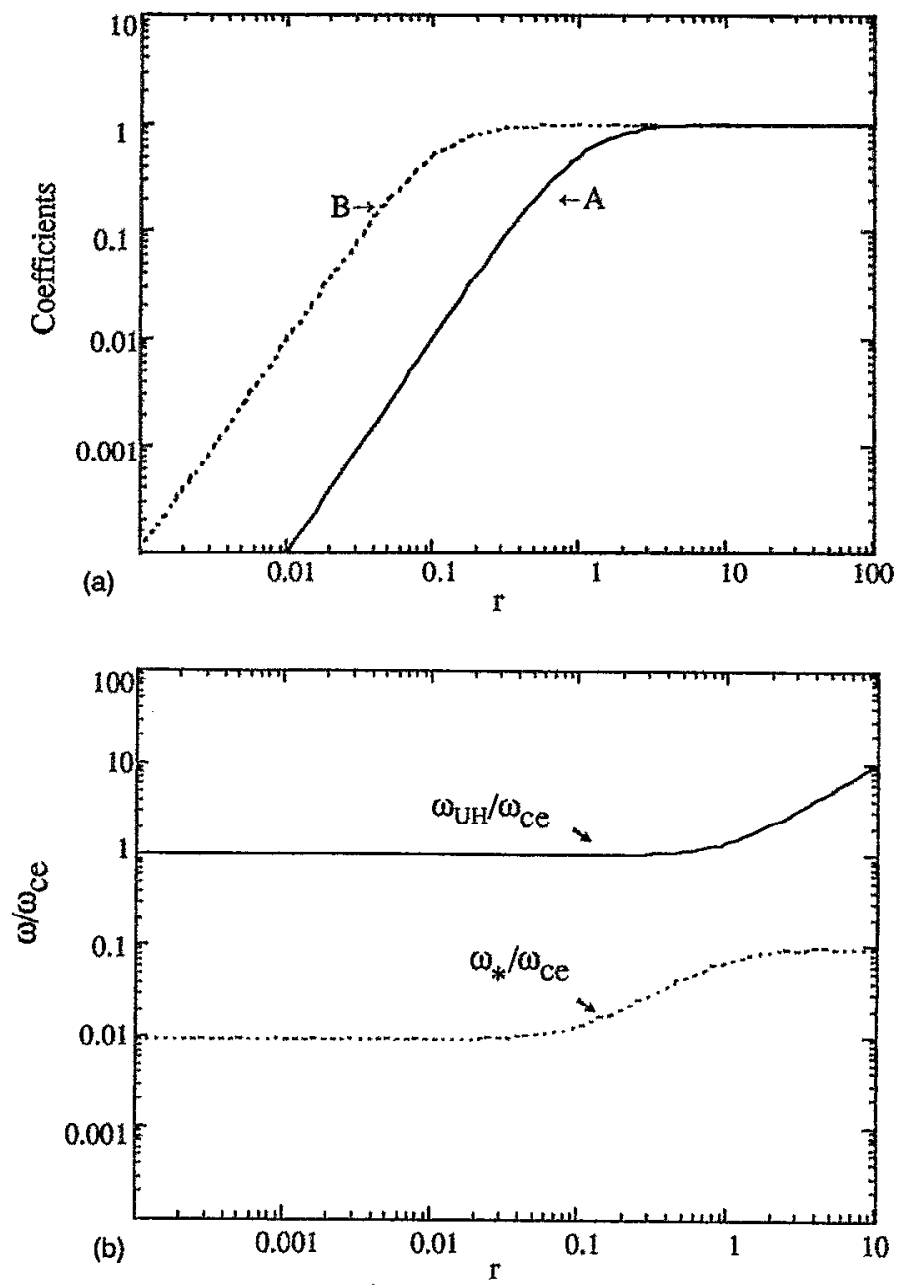

FIG. 1. Plots of important parameters obtained for the elcctrostatic solution. (a) Amplitude coefficients $A=\omega_{p}^{2} / \omega_{\mathrm{UH}}^{2}$ and $B=\omega_{\rho}^{2} /\left(\omega_{p}^{2}+\omega_{\mathrm{LH}}^{2}\right)$ for the electrostatic polarization field plotted as a function of $r=\omega_{p e} / \omega_{c e}$ (b) Frequency modes $\omega_{\mathrm{UH}} / \omega_{c e}$ and $\omega_{\bullet} / \omega_{c e}=\sqrt{\omega_{\mathrm{LH}}^{2}\left(\omega_{p}^{2}+\omega_{\mathrm{LH}}^{2}\right) / \omega_{\mathrm{UH}}^{2} \omega_{c e}^{2}}$ for the electrostatic polarization field plotted as a function of $r$. An ion-toelectron mass ratio of 100 is assumed in these plots.

tremes, with oscillation frequencies ranging from the ion cyclotron frequency through the lower-hybrid range and up to the upper-hybrid frequency. To further elucidate the temporal behavior of the polarization field, we have plotted the solution, Eq. (12), for three values of the parameter $r(=0.01,1,100)$ with high time resolution and for a short period of time in Figs. 2(a)-2(c) and with low time resolution and for an extended period of time in Figs. 2(d)$2(f)$. The changes in field amplitude and oscillation frequency with $r$ are clearly seen in these plots.

It is instructive to examine the partitioning of momentum and energy between the fields and the plasma. For this purpose, we introduce the parameter $\gamma_{m}\left(\gamma_{e}\right)$, defined to be the ratio of mean field momentum (energy) density in the $y$ direction to initial plasma momentum (energy) density in the $y$ direction, i.e.,

$$
\gamma_{m}=\frac{\left\langle E_{x}\right\rangle B_{0} / 4 \pi c}{n\left(m_{e}+m_{i}\right) V_{0}}=\frac{\omega_{c i}^{2}}{\omega_{p i}^{2}\left(1+m_{e} / m_{i}\right)} B
$$



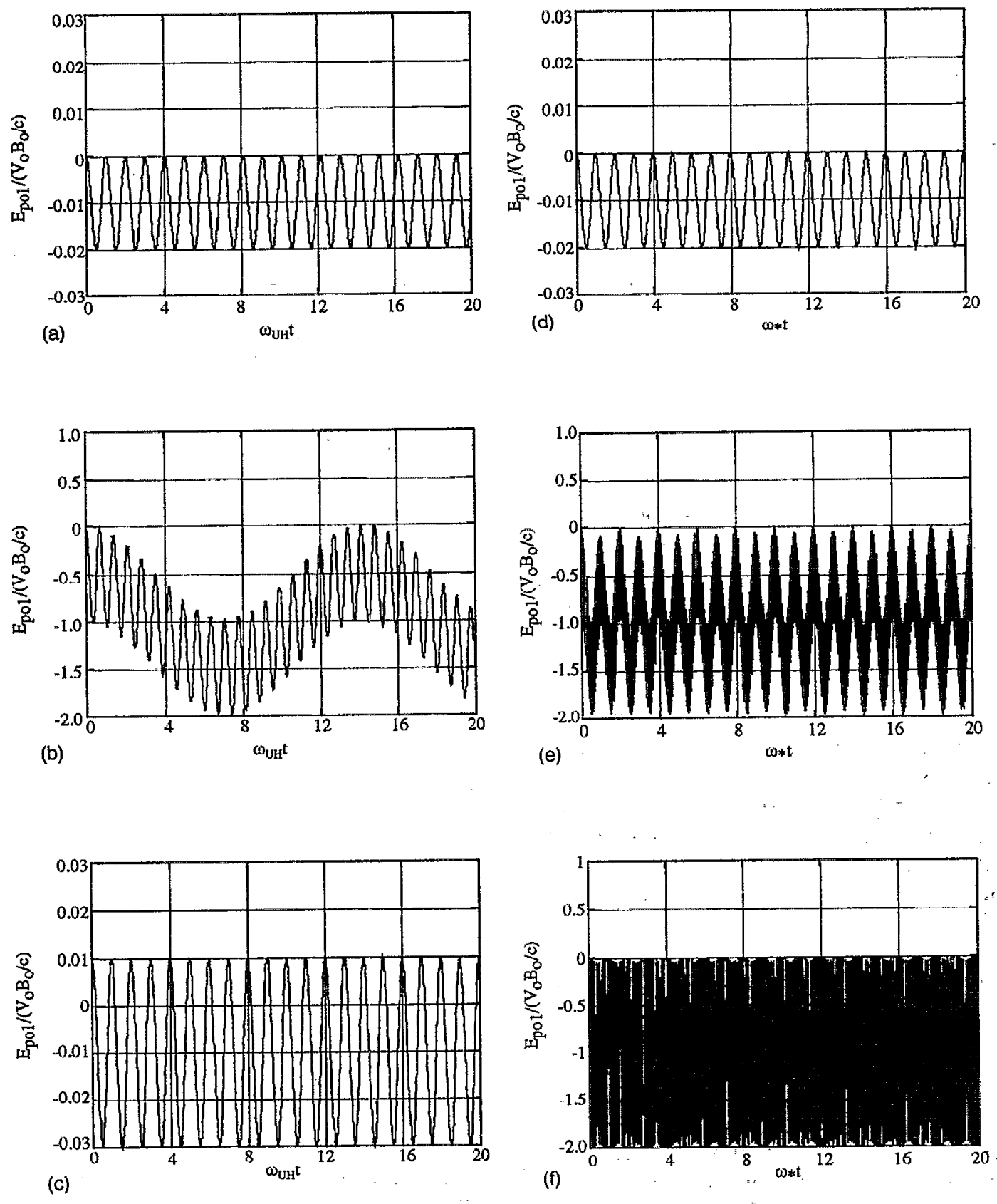

FIG. 2. Plots of the electrostatic polarization field as a function of time. (a)-(c) Amplitude of the electrostatic polarization electric field plotted as a function of time normalized to the upper-hybrid period, i.e., $\tau=\omega_{\mathrm{UH}} t$, for values of the parameter $r=0.01,1$, and 100, respectively. (d)-(f) Amplitude of the electrostatic polarization electric field plotted as a function of time normalized to lower-hybrid and ion cyclotron time scales, i.e., $\tau=\omega_{\bullet} t$, for values of the parameter $r=0.01,1$, and 100 , respectively. An ion-to-electron mass ratio of 100 is assumed in these plots.

$$
\begin{aligned}
\gamma_{e} & =\frac{\left\langle E_{x}^{2}\right\rangle / 8 \pi}{n_{e}\left(m_{e}+m_{i}\right) V_{0}^{2} / 2} \\
& =\frac{\omega_{c i}^{2} \cdots}{\omega_{p i}^{2}\left(1+m_{e} / m_{i}\right)}\left(A^{2}+\frac{3 B^{2}}{2}-A B\right),
\end{aligned}
$$

where $A$ and $B$ are the coefficients defined above and the angle brackets denote a time average over many oscillation periods. The parameters $\gamma_{m}\left(\gamma_{e}\right)$ are plotted in Fig. 3 as a function of $r$ for an ion-to-electron mass ratio of 100. In the high density limit (large $r$ ), we find that the average momentum (energy) density stored in the fields is small com- pared to the initial plasma momentum (energy) density and that $\gamma_{m} \approx 2 \gamma_{e} / 3$. Thus the plasma moves across the background magnetic field nearly unimpeded as discussèd above. In the low density limit (small $r$ ), $\gamma_{m}$ approaches unity while $\gamma_{e}$ is small and decreases with decreasing $r$. In this scenario, nearly the entire initial momentum of the plasma is stored in the fields while the plasma simply gyrates about an equilibrium position, retaining most of its energy. For intermediate values of $r$, we find that the energy density stored in the fields maximizes at $r \approx 0.1$ while the momentum density stored in the fields decreases mono- 


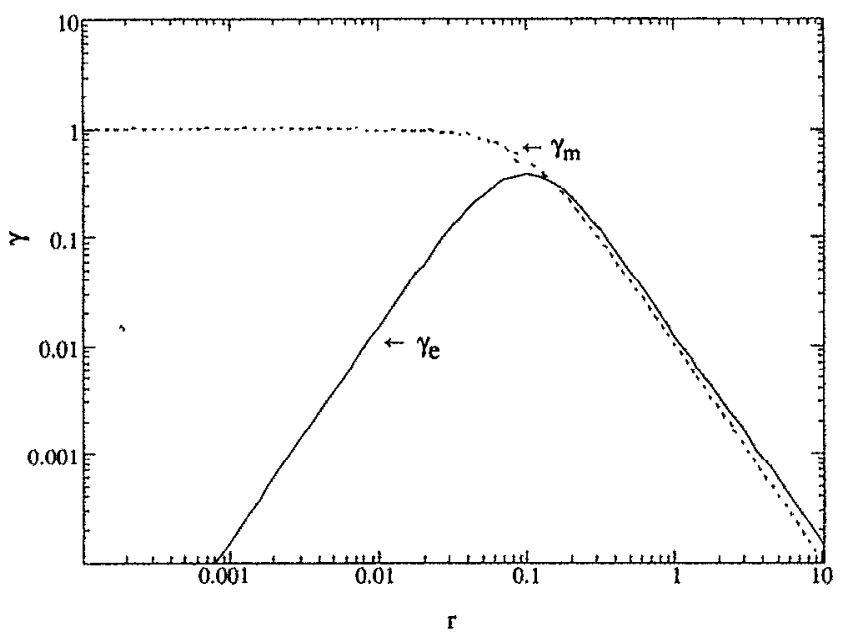

FIG. 3. Ratios $\gamma_{m}$ of the mean field momentum density in the beam direction to the initial plasma momentum in the beam direction and $\gamma_{e}$ of the mean field energy density in the beam direction to the initial plasma kinetic energy density in the beam direction plotted as a function of the parameter $r=\omega_{p e} / \omega_{c e}$ An ion-to-electron mass ratio of 100 is assumed in these plots.

tonically with $r$. This result can be understood by examining the ratio of the ion Debye length $\left(\lambda_{\mathrm{D} i}\right)$ to the ion gyroradius $\left(\rho_{i}\right)$ as a function of $r$. Let $\alpha=\lambda_{\mathrm{D} i} / \rho_{i}$ $=\sqrt{m_{e} / m_{i}} 1 / r$. For large $r, \alpha$ is small and the amount of energy and momentum stored in the polarization field is also small, simply because the ions can only gyrate a tiny fraction of their gyro-orbit before the polarization field is able to stop their motion perpendicular to the initial direction of motion. In this regime, the ions exhibit oscillatory orbits in the plane perpendicular to the magnetic field as depicted in Fig. 4(a). We expect the maximum energy to be stored in the polarization field when $\alpha=1$ so that the full gyromotion of the ion goes into charge separation and indeed $\gamma_{e}$ maximizes at a value of approximately $40 \%$ when $r \approx 0.1$, corresponding to $\alpha=1$ for an ion-to-electron mass ratio of 100 . In this latter regime, the ions exhibit partial gyro-orbits and $E_{\text {pol }} \times B_{0}$ drifts, as depicted in Fig. 4(b). As $r$ decreases further, the Debye length becomes less than an ion gyroradius and a smaller fraction of the initial plasma energy is stored in the polarization field. At the same time, the ions can now gyrate "freely" and therefore more of the initial momentum must be stored in the field. In this regime, the ions exhibit gyromotion primarily, as depicted in Fig. 4(c).

Finally, we note that, in the limit of our approximations (discussed in Paper I), the electrostatic resulis obtained above apply independently to every position in the plasma, i.e., there is no communication across the plasma. Thus a differential braking of the plasma cloud occurs in which the denser central portions continue unimpeded across the magnetic field while the less dense outer layers become trapped and simply gyrate around the field. Moreover, in the absence of radiation fields, the $y$ components of the Maxwell stress tensor and the Poynting flux are zero so that the total plasma and field momentum and energy den-
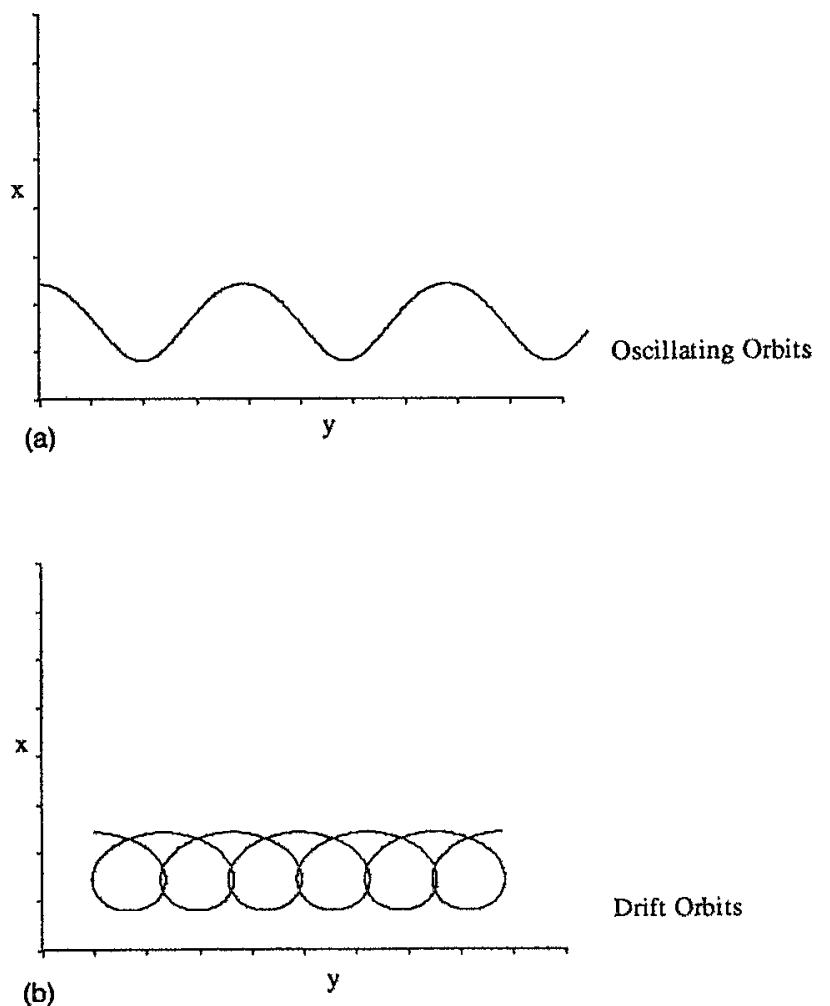

Drift Orbits

(b)

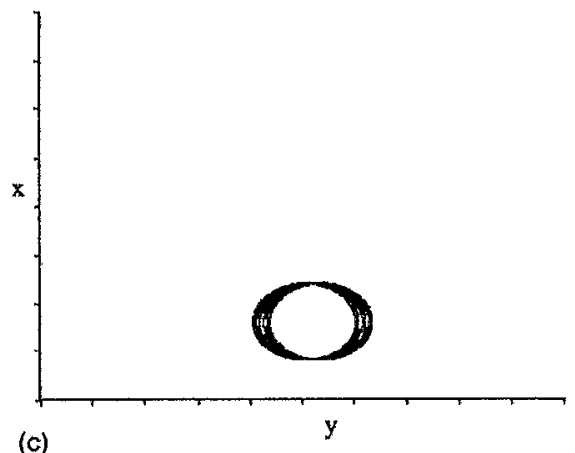

Gyro Orbits

(c)

FIG. 4. Sketch of ion orbits in a background magnetic field for three plasma density regimes corresponding to large (a), intermediate (b), and small (c) values of the parameter $r=\omega_{p e} / \omega_{c e}$

sities must remain constant at every position for all time [see Eqs. (34) and (35) of Paper I]. As a result, the electrostatic solution is characterized by a constant local exchange of momentum and energy between the fields and the plasma, driven and maintained by the inertial separation of charge.

Because of the presence of oscillating currents, the plasma will, in general, radiate coherently. The role of the radiation field will be in a sense to provide a viscous transport of momentum that will attempt to establish a certain level of communication and cohesiveness across the plasma cloud. In addition, however, some of the radiated energy and momentum will escape and the question is how much 
and what is the ultimate fate of the plasma cloud. These issues are addressed quantitatively in the following section.

\section{ELECTROMAGNETIC RESULTS}

To zeroth order in the Hermite polynomial expansion, the final solution obtained in Paper I for the normalized (to $V_{0} B_{0} / c$ ) electric field is given by

$$
\begin{aligned}
\widetilde{\mathbf{E}}(s, x, z)= & e^{-x^{2} / D^{2}}\left[\int _ { - \infty } ^ { z } d z ^ { \prime } \frac { s } { c ^ { 2 } } \left(\mathbf{e}_{0} \alpha_{0}\left(s, z^{\prime}\right) e^{i \int_{z^{\prime}}^{z}, k_{z^{\prime \prime}}^{0}} d z^{\prime \prime}\right.\right. \\
& \left.\left.+\mathbf{e}_{1} \alpha_{1}\left(s, z^{\prime}\right) e^{i \int_{z^{\prime}}^{z} k_{z^{\prime \prime}}^{1} d z^{\prime \prime}}\right)\right] \\
& +e^{-x^{2} / D^{2}}\left[\int _ { z } ^ { \infty } d z ^ { \prime } \frac { s } { c ^ { 2 } } \left(\mathbf{e}_{0} \alpha_{0}\left(s, z^{\prime}\right) e^{-i \int_{z^{\prime}}^{z} k_{z^{\prime \prime}}^{0} d z^{\prime \prime}}\right.\right. \\
& \left.\left.+\mathbf{e}_{1} \alpha_{1}\left(s, z^{\prime}\right) e^{-i \int_{z^{\prime}}^{z} k_{z^{\prime \prime}}^{1}, z^{\prime \prime}}\right)\right],
\end{aligned}
$$

where the coefficients $\alpha_{0}$ and $\alpha_{1}$ are defined as

$$
\begin{aligned}
& \alpha_{0}=\frac{s^{2} \beta_{2}^{2}+\beta\left(c^{2} / D^{2}+\sqrt{c^{4} / D^{4}-s^{4} \beta_{2}^{2}}\right)}{4 i k_{z^{\prime}}^{0} \sqrt{c^{4} / D^{4}-s^{4} \beta_{2}^{2}}}, \\
& \alpha_{1}=-\frac{s^{2} \beta_{2}^{2}+\beta\left(c^{2} / D^{2}-\sqrt{c^{4} / D^{4}-s^{4} \beta_{2}^{2}}\right)}{4 i k_{z^{\prime}}^{1} \sqrt{c^{4} / D^{4}-s^{4} \beta_{2}^{2}}},
\end{aligned}
$$

the two eigenvalues $k_{z}^{0}$ and $k_{z}^{1}$ derived in Paper I are

$$
\begin{aligned}
& c^{2}\left(k_{z}^{0}\right)^{2}=-\left(s^{2}(1+\beta)+\frac{c^{2}}{D^{2}}\right)+\sqrt{\frac{c^{4}}{D^{4}}-s^{4} \beta_{2}^{2}}, \\
& c^{2}\left(k_{z}^{1}\right)^{2}=-\left(s^{2}(1+\beta)+\frac{c^{2}}{D^{2}}\right)-\sqrt{\frac{c^{4}}{D^{4}}-s^{4} \beta_{2}^{2}},
\end{aligned}
$$

with $\beta$ and $\beta_{2}$ functions of $z$ given by

$$
\begin{aligned}
& \beta=4 \pi \sigma_{\mathrm{P}} e^{-z^{2} / d^{2}} / s, \\
& \beta_{2}=4 \pi \sigma_{\mathrm{H}} e^{-z^{2} / d^{2}} / s,
\end{aligned}
$$

$s$ is the Laplace transform variable, $D(d)$ is the $1 / e$ halfwidth of the plasma cloud in the $x(z)$ direction, and $\sigma_{\mathrm{P}}$ and $\sigma_{\mathrm{H}}$ are the Pedersen and Hall conductivities, respectively, defined by Eqs. (13) and (14) of Paper I. The corresponding eigenvectors for these two modes are given by

$$
\begin{aligned}
& \mathrm{e}^{0}=\left[\begin{array}{c}
1 \\
\frac{s^{2} \beta_{2}}{c^{2} / D^{2}+\sqrt{c^{4} / D^{4}-s^{4} \beta_{2}^{2}}} \\
0
\end{array}\right], \\
& \mathrm{e}^{1}=\left[\begin{array}{c}
1 \\
\frac{s^{2} \beta_{2}}{c^{2} / D^{2}-\sqrt{c^{4} / D^{4}-s^{4} \beta_{2}^{2}}} \\
0
\end{array}\right],
\end{aligned}
$$

and the inverse Fourier transform in $x$ has already been performed. The integrals in Eq. (15) are performed numerically as is the inverse Laplace transform using a technique described by Dubner and $\mathrm{Abate}^{9}$ for conversion to fast Fourier transforms.
TABLE I. Magnetospheric release parameters one second after detonation.

\begin{tabular}{cc}
\hline Total barium mass released & $1.5 \mathrm{~kg}$ \\
Total barium mass vaporized & $0.6 \mathrm{~kg}$ \\
Total number of barium neutrals & $2.62 \times 10^{24}$ \\
Estimated plasma density & $1 \times 10^{7} \mathrm{~cm}^{-3}$ \\
Dimension along ambient magnetic field & $1 \mathrm{~km}$ \\
Dimension perpendicular to magnetic field & $1 \mathrm{~km}$ \\
Beam velocity & $6.8 \mathrm{~km} / \mathrm{sec}$ \\
Ambient magnetic field strength & $0.08 \mathrm{G}$ \\
\hline
\end{tabular}

To illustrate the properties of our final solution, we have selected parameters corresponding to those attained in a magnetospheric barium release performed as part of the CRRES campaign at a geocentric distance of two Earth radii. The release parameters are summarized in $\mathrm{Ta}$ ble I, which also lists estimates for the plasma density $1 \mathrm{sec}$ after the release and for the background magnetic field strength. We do not intend to provide a detailed comparison between theory and experiment in this particular paper simply because the data analysis has not been performed. It is not necessary therefore to elaborate on the experiment. We will, however, examine the theoretical results quantitatively and provide scaling laws as well as a prediction for the momentum loss time scale. In order to reduce the computational time, we have made two simplifying approximations; namely, that the ion mass is only 100 times the electron mass and that the cloud dimension along the magnetic field is small $(=0.06 \mathrm{~km})$. The former brings the ion dynamics into the computation sooner while the latter reduces the amount of time for the radiation to propagate out of the plasma cloud. Because we are solving a linear set of equations, the effects of interest are readily scaled to experimental conditions. The actual parameters used in our calculation are summarized in Table II. We divide our discussion into six subsections dealing with the dispersion relations for the normal modes of our solution, the results for the $x$ and $y$ electric fields presented on electron plasma period, electron cyclotron, and ion cyclotron time scales, and finally a discussion of the partitioning of momentum and energy between the electromagnetic fields and the plasma.

TABLE II. Calculation parameters.

\begin{tabular}{cc}
\hline \hline Beam velocity $\left(V_{0}\right)$ & $6.8 \mathrm{~km} / \mathrm{sec}$ \\
Ambient magnetic field strength $\left(B_{0}\right)$ & $0.08 \mathrm{G}$ \\
Dimension along magnetic field $(d)$ & $0.06 \mathrm{~km}$ \\
Dimension perpendicular to magnetic field $(D)$ & $1 \mathrm{~km}$ \\
Plasma density in center of plasma cloud $\left(n_{0}\right)$ & $6.0 \times 10^{6} \mathrm{~cm}^{-3}$ \\
Ion/electron mass ratio $\left(m_{i} / m_{e}\right)$ & 100 \\
Electron plasma frequency in cloud center $\left(f_{p e}\right)$ & $2.2 \times 10^{7} \mathrm{sec}^{-1}$ \\
Electron gyrofrequency $\left(f_{c e}\right)$ & $2.2 \times 10^{5} \mathrm{sec}^{-1}$ \\
Upper-hybrid frequency $\left(f_{\mathrm{UH}}\right)$ & $2.2 \times 10^{7} \mathrm{sec}^{-1}$ \\
Ion gyrofrequency $\left(f_{c i}\right)$ & $2.2 \times 10^{3} \mathrm{sec}^{-1}$ \\
Lower-hybrid frequency $\left(f_{\mathrm{LH}}\right)$ & $2.2 \times 10^{4} \mathrm{sec}^{-1}$ \\
\hline
\end{tabular}




\section{A. Dispersion relations}

The dispersion relations for the parameters given in Table II are shown in Figs. 5(a)-5(c) at three positions along the magnetic field. Plotted in these figures is the index of refraction squared $\left(=c^{2} k_{z}^{2} / \omega^{2}\right)$ for each of two normal modes (right-hand circularly polarized waves and left-hand circularly polarized waves as discussed in Paper I) as a function of frequency $\omega$, where we have substituted $i \omega$ for the Laplace transform variable $s$. In these figures, the index of refraction squared is normalized to a stated value and the $\log$ of this quantity is plotted along the ordinate. In addition, the sign of this quantity is preserved so that values that fall below zero correspond to evanescent, nonpropagating waves. As discussed in Paper I, the dispersion relations take on the usual form expected for right-hand and left-hand circularly polarized waves in a cold plasma imbedded in a background magnetic field (e.g., Krall and Trivelpiece ${ }^{10}$ ). At high frequencies, the left-hand and right-hand modes are both dispersive but become evanescent at frequencies below $\omega_{1}=\left(\omega_{c e} / 2\right)\left(\sqrt{1+4 \omega_{p e}^{2} / \omega_{c e}^{2}}\right.$ - 1) for the left-hand mode and $\omega_{2}=\left(\omega_{\mathrm{ce}} / 2\right)$ $\times\left(\sqrt{1+4 \omega_{p e}^{2} / \omega_{c e}^{2}}+1\right)$ for the right-hand mode, where $\omega_{p e}$ is the electron plasma frequency and $\omega_{c e}$ is the electron cyclotron frequency. The electron and ion cyclotron resonances appear in the dispersion plots as do electron and ion cyclotron waves, whistler waves, and Alfvén waves at low frequencies. In the limit that the plasma cloud is large and the plasma density is sufficiently large, i.e., $\left|\omega^{4} \beta_{2}^{2}\right| \gg c^{4} / D^{4}$, expressions for $\omega_{1}$ and $\omega_{2}$ applicable at low frequencies can be obtained; namely,

$$
\begin{aligned}
\omega_{1}= & \frac{\omega_{c e}}{2}\left(\sqrt{1+\frac{4 \omega_{p e}^{2}\left(1+\omega_{\mathrm{LH}}^{2} / \omega_{c e}^{2}\right)+\omega_{c i}^{2}+2 \omega_{\mathrm{LH}}^{2}}{\omega_{c e}^{2}}}\right. \\
& \left.-\left(1-\omega_{c i} / \omega_{c e}\right)\right), \\
\omega_{2}= & \frac{\omega_{c e}}{2}\left(\sqrt{1+\frac{4 \omega_{p e}^{2}\left(1+\omega_{\mathrm{LH}}^{2} / \omega_{c e}^{2}\right)+\omega_{c i}^{2}+2 \omega_{\mathrm{LH}}^{2}}{\omega_{c e}^{2}}}\right. \\
& \left.+\left(1-\omega_{c i} / \omega_{c e}\right)\right),
\end{aligned}
$$

where $\omega_{\mathrm{LH}}\left(=\sqrt{\omega_{c r} \omega_{c e}}\right)$ is the lower-hybrid frequency. Thus, as we move to higher $z$ in the cloud, the plasma density decreases and we see the high frequency cutoffs of $\omega_{1}$ and $\omega_{2}$ shift to lower frequencies with $\omega_{1}$ approaching $\omega_{c i}$ and $\omega_{2}$ approaching $\omega_{c e}$. In addition, we find the lowfrequency cutoff of the right-hand mode appearing in the vicinity of $\omega_{c i}$ at the outer edge of the plasma. This lowfrequency cutoff increases as a function of $z$ from zero frequency to the value $\left(\sim 2.5 \omega_{c i}\right)$ obtained at $z / d=3.0$. For electron plasma frequencies much larger than the cutoff frequency, we have

$$
\omega_{\text {cutoff }} \approx \frac{c}{D}\left(\frac{\omega_{c e} c}{\omega_{p e}^{2} D}-\frac{\omega_{\mathrm{LH}}}{\omega_{p e}}\right)
$$

As the electron density increases, we see that $\omega_{\text {cutoff }}$ decreases.

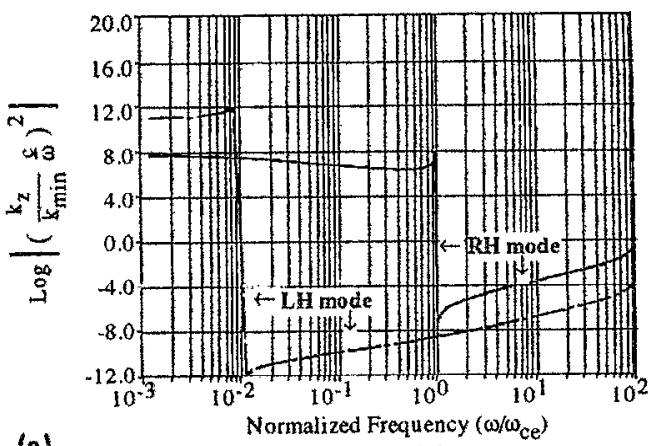

(a)

$$
\mathrm{Z} / \mathrm{d}=0.0
$$

$K_{\min }^{R}=1.98 \times 10^{-2}$

$\mathrm{K}_{\mathrm{atu}=1}^{\mathrm{min}}=1.09 \times 10^{-5}$

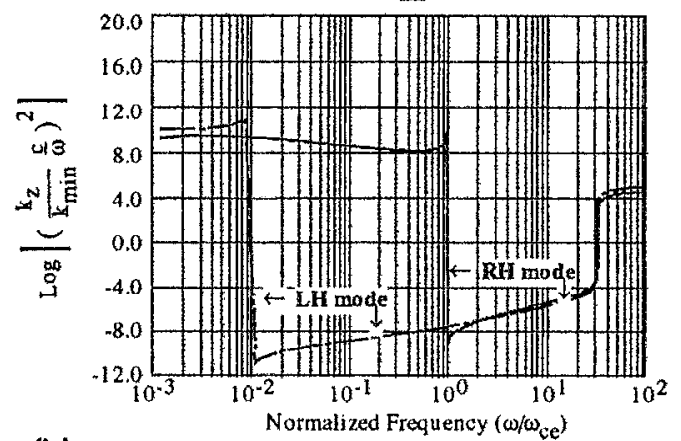

(b)

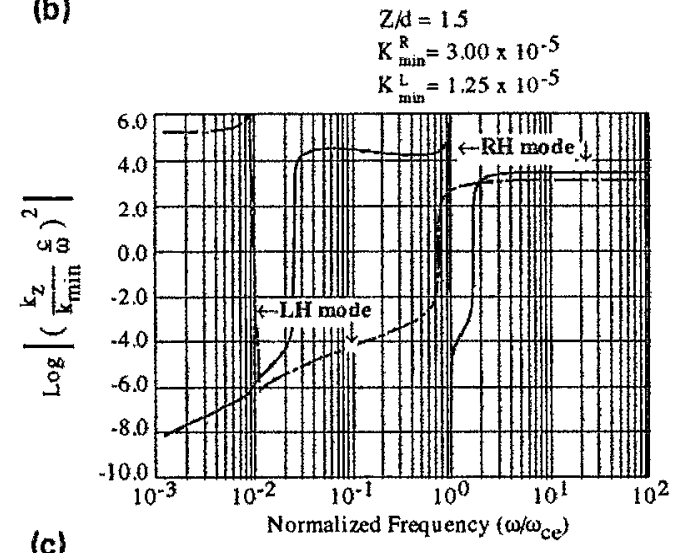

(c)

$$
\begin{aligned}
& \mathrm{Z} / \mathrm{A}=3.0 \\
& \mathrm{~K}_{\min }^{\mathrm{R}}=3.93 \times 10^{-4} \\
& \mathrm{~K}_{\min }^{\mathrm{L}}=8.73 \times 10^{-4}
\end{aligned}
$$

FIG. 5. The index of refraction squared $\left(=c^{2} k_{z}^{2} / \omega^{2}\right)$ for the two eigenmodes associated with the zeroth-order solution is plotted as a function of frequency $\omega$ at three positions along the $z$ dimension of the cloud (a)-(c). In these figures, the index of refraction squared is normalized to a stated value and the log of this quantity is plotted along the ordinate. In addition, the sign of this quantity is preserved so that values that fall below zero correspond to evanescent, nonpropagating waves. For these particular plots, we have $\omega_{p e}(z=0)=100 \omega_{c e} \omega_{c e}=100 \omega_{c i}$ and $D=1 \mathrm{~km}$. In the limit of very large plasma clouds $\left(c / D<\omega_{c i}\right)$, the dispersion relations take on the usual form expected for right-hand and left-hand circularly polarized waves in a cold plasma imbedded in a background magnetic field. At high frequencies, the left-hand and right-hand modes are both dispersive but become evanescent at frequencies below $\omega_{1}\left\{=1 / 2 \omega_{c e}\left[\left(1+4 \omega_{p e}^{2} / \omega_{c e}^{2}\right)-1\right]\right\}$ for the left-hand mode and $\omega_{2}\left\{=1 / 2 \omega_{c e}\left[\left(1+4 \omega_{p e}^{2} / \omega_{c e}^{2}\right)+1\right]\right\}$ for the right-hand mode. The electron and ion cyclotron resonances appear in the dispersion plots as do electron and ion cyclotron waves, whistler waves, and Alfvén waves at low frequencies. As we move to higher $z$ in the cloud, the plasma density decreases and we see $\omega_{1}$ and $\omega_{2}$ shift to lower frequencies. In addition, an evanescent cutof is introduced at low frequencies in the vicinity of $\omega_{c i}$ as a result of the finite dimension of the radiating plasma antenna. 
As noted in Paper I; the denominator inside the $z^{\prime}$ integral of Eq. (15) has zeros at the electron and ion cyclotron frequencies and at frequencies where $k_{z}$ goes to zero. These frequencies correspond to the natural modes of oscillation of the plasma and are frequencies at which the cloud will radiate. The right-hand radiation is associated with currents derived from the electron motion while the left-hand radiation is derived from the ion motion. Based on the dispersion plots, we expect to see right-hand and left-hand radiation emanating from the cloud throughout the frequency range $\omega_{c e}<\omega<\omega_{2}(z=0)$. With decreasing $\omega_{p e}$, low-frequency right-hand oscillations occur from zero frequency to somewhat above $\omega_{c l}$ while left-hand waves are generated below $\omega_{c e}$. While these frequencies represent the normal modes of oscillation of the plasma cloud, it is clear that radiation damping will broaden the frequency content of the current systems and the self-consistent radiation fields. In a manner analogous to an $L C R$ circuit in which the resistance $(R)$ of the circuit acts to damp the natural oscillation of energy defined by the circuit inductance $(L)$ and capacitance $(C)$, the radiation field that flows out of the plasma cloud acts to damp the natural modes of oscillation and the impulse response of the system is therefore characterized by a spectrum in which the energy associated with the normal modes of the system is spread over a finite but broad frequency range. Thus we expect to see radiation occurring over the entire frequency range permitted by the initial impulsive development of the plasma release. In addition, we expect to see most of the energy in the lower frequencies approaching the ion cyclotron frequency simply because most of the energy is stored in the ions.

\section{B. Solution on electron plasma period time scales}

With this basic interpretation of the spectral content of our solution, we now present the temporal results obtained by taking the inverse Laplace transform of Eq. (15). Figures $6(a)-6(c)$ contain plots of the $x$ component of the clectric field, normalized to $V_{0} B_{0} / c$, as a function of time, normalized to the minimum cloud electron plasma period, at three locations in the cloud; namely, $x=0, z=0 ; x=0$, $z=1.5 d$; and $x=0, z=3.0 d$; where $x=0, z=0$ is a position in the center of the cloud. Figures $6(d)-6(f)$ are the corresponding Fourier transforms of the $x$ electric fields. For this set of results, the Nyquist frequency was chosen to be approximately six times the maximum plasma frequency of the cloud and the calculations were carried out to only 100 corresponding electron plasma periods or one electron cyclotron period. At this point in our discussion, we are only examining the early evolution of the plasma cloud that is dominated by the electron motion. Below, we will present results out to many electron and ion cyclotron periods. Similar plots for the $y$ component of the electric field are shown in Figs. $7(\mathrm{a})-7$ (f). The $x$ component of the electric field in the center of the cloud oscillates about a mean value equal to the polarization electric field $\left(=-V_{0} B_{0} / c\right)$ necessary to cause the plasma to drift with velocity $V_{0}$ across the magnetic field. Though not easily discernible from Fig. 6(d), the spectrum contains two peaks at frequencies corresponding to $\omega_{1}$ and $\omega_{2}$, separated by the electron cyclo- tron frequency, and the beating of these two frequency modes can be seen in the temporal plot. The amplitude of the oscillations is damped as the radiation field carries energy out of the center of the cloud. The effect of radiation damping is also seen in the spectral plot as a broadening of the normal modes. As one moves to the outer layers of the cloud, the plasma density decreases and the steady-state component of the $x$ electric field decreases in amplitude simply because the plasma cannot supply enough charge to maintain the necessary polarization electric field. The latter statement is made clearer by realizing that, as the electron plasma Debye length exceeds the electron gyroradius (decreasing density), a smaller fraction of the electron kinetic energy is stored in the polarization electric field. Gradually, the electron motion becomes dominated by the background magnetic field and a transition from simple harmonic oscillations to $E \times B$ drifting and electron gyromotion occurs as the Debye length approaches and eventually exceeds the gyroradius (i.e., as the electron density decreases). The fact that $\left\langle E_{x}\right\rangle=-V_{0} B_{0} / c$ does not imply $E \times B$ drifting at the initial velocity $V_{0}$, but rather that harmonic oscillatory motion is occurring, which allows the electrons on average to proceed at a velocity $V_{0}$ across the magnetic field. Indeed, $E \times B$ drifting only occurs in regions of the plasma where the average field is less than $V_{0} B_{0} / c$ in magnitude and in these regions the electrons are unable to keep up with the ions. Clearly, these remarks only apply to situations in which the electric field is formed self-consistently by the plasma. Thus the electrons in the outer layers of the plasma are slowing down and producing a current in the $y$ direction. For a finite plasma in the direction of motion, this effect would manifest itself as a $y$-component electric field and as a shearing off of the outer layers of the plasma.

The propagation of high-frequency radiation from the central part of the plasma cloud through the outer layers is also evident in Figs. 6(a)-6(f). Notice that the spectrum broadens in the outer layers of the plasma as radiation from the normal modes generated in the inner layers propagates outward. In addition, the radiation field in the outer layers appears as a chirp going from low frequencies to high frequencies consistent with the arrival time of various contributions from the inner plasma layers. Similar effects are seen in the $y$ component of the electric field, Figs. $7(a)-7(f)$, except of course that there is no steady-state value because this component is strictly an induction field. Note that the beating of the right-hand and left-hand modes is out of phase by half an electron gyroperiod relative to the $x$-component electric field as expected.

\section{Solution on electron cyciotron period time scales}

Similar results for the $x$ component of the electric field as a function of time, normalized to an electron cyclotron period, for 100 cycles or one ion gyroperiod are shown in Figs. 8(a)-8(c) with the corresponding Fourier transforms shown in Figs. 8(d)-8(f). A complementary set of plots for the $y$ component of the electric field are presented in Figs. 9(a)-9(f). With a Nyquist frequency of only six times the electron gyrofrequency, we note that the details 

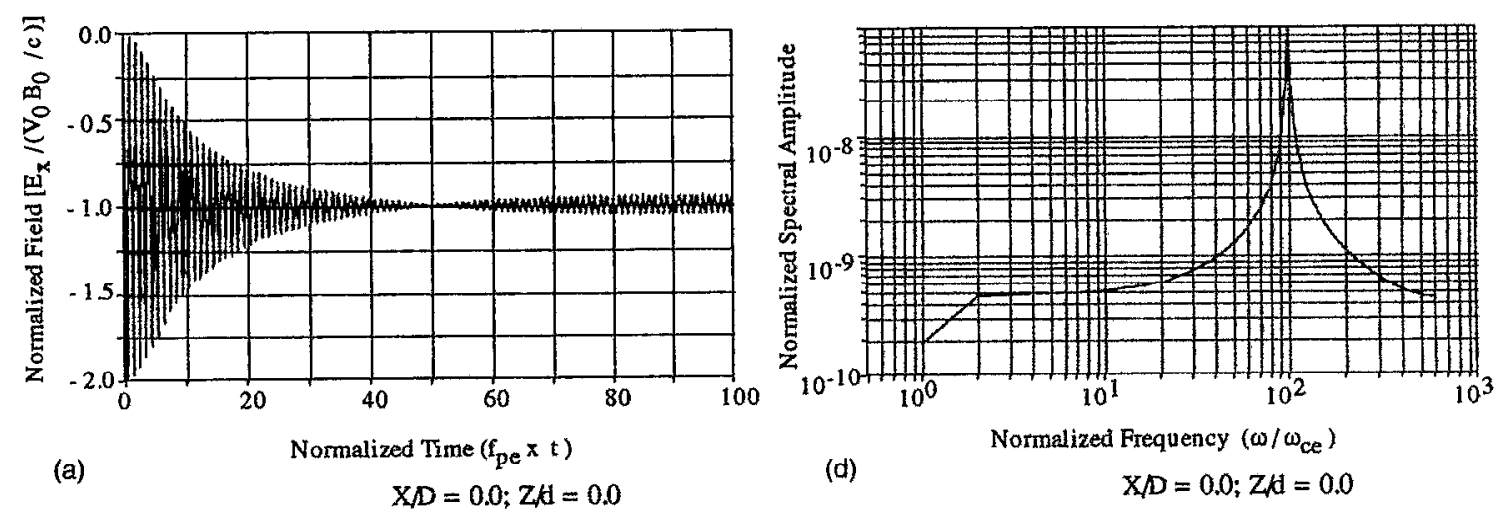

(d)

$$
\mathrm{XD}=0.0 ; \mathrm{Zdd}=0.0
$$

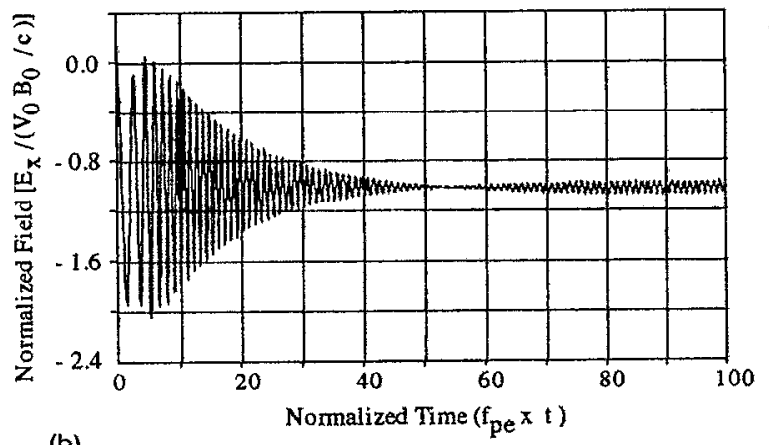

(b)

$X D=0.0 ; Z / d=1.5$

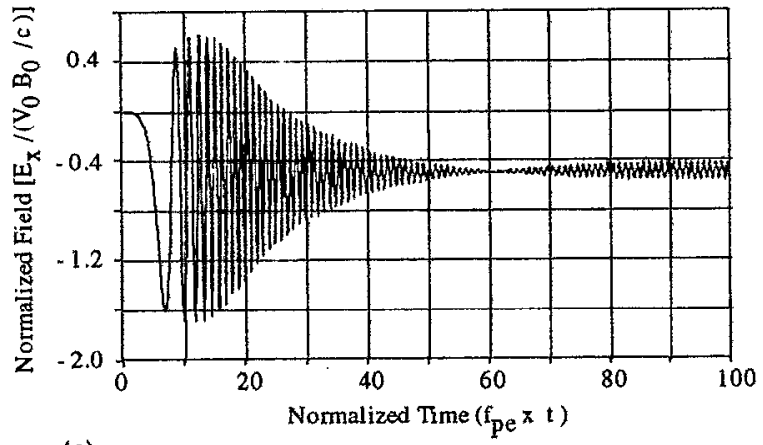

(c)

$X / D=0.0 ; Z / A=3.0$

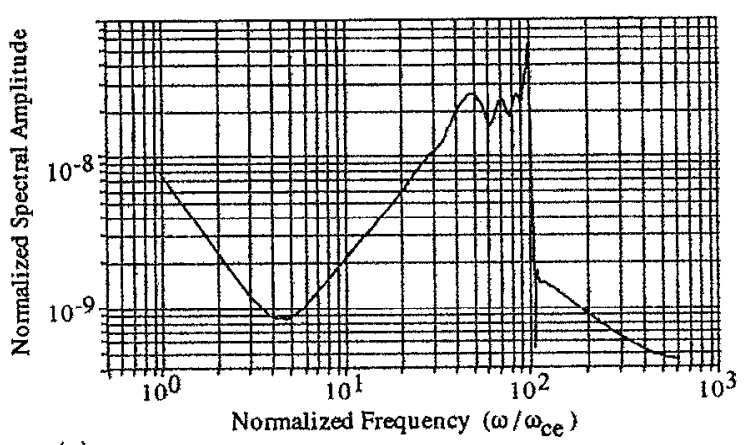

$X D=0.0 ; Z / A=1.5$

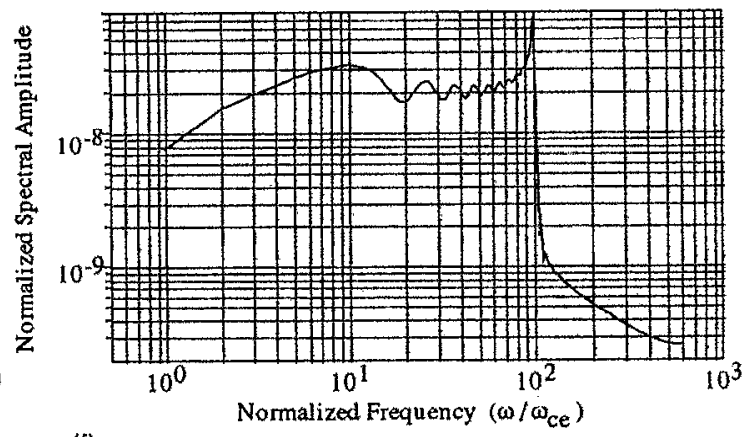

(f)

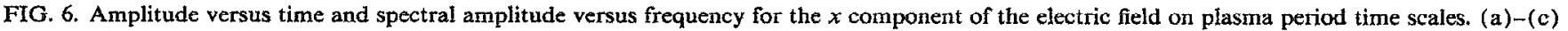

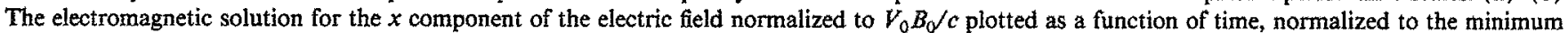

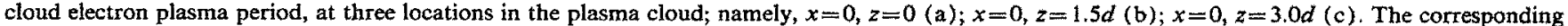

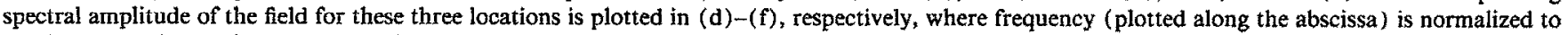
an electron cyclotron frequency and the Nyquist frequency was chosen to be six times the peak cloud electron plasma frequency.

of the dynamics discussed above will be lost and only the low-frequency oscillations are resolved. In the center of the plasma cloud, the $x$ component of the electric field achieves a steady-state value of $-V_{0} B_{0} / c$ almost instantaneously followed by the development of a large-amplitude pulse that chirps from high frequencies to low frequencies and that causes the polarization electric field to decay to zero. This pulse develops on lower-hybrid time scales and has a right-hand sense and frequency content consistent with a whistler wave. The spectral amplitude increases with decreasing frequency and has an oscillation with a period that is consistent with the superposition of two pulses delayed by approximately ten electron cyclotron periods. A temporal sequence of spatial contour plots, which will be discussed below, indicates that the pulse initially forms in the outer layers of the plasma and propagates through the 


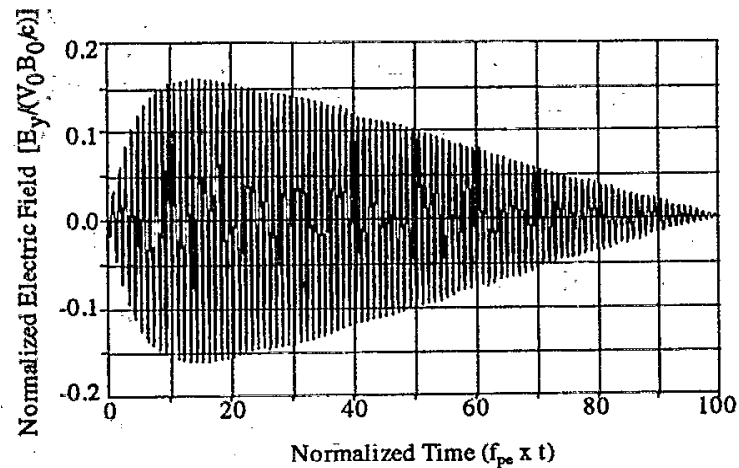

(a)

$$
\mathrm{X} / \mathrm{d}=0.0 ; \mathrm{Z} / \mathrm{d}=0.0
$$

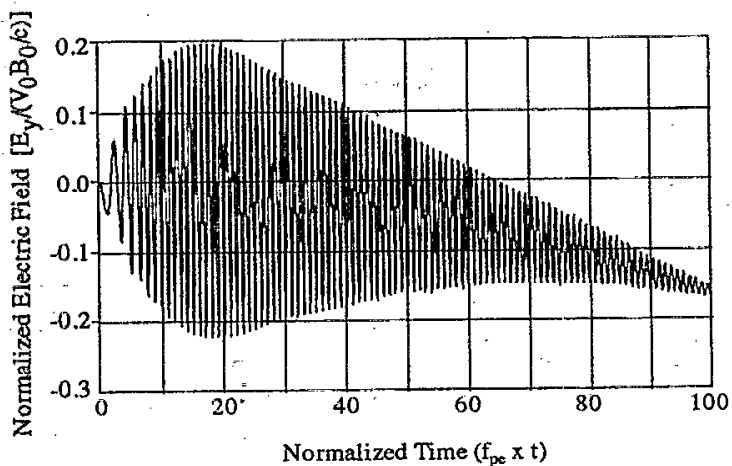

(b)

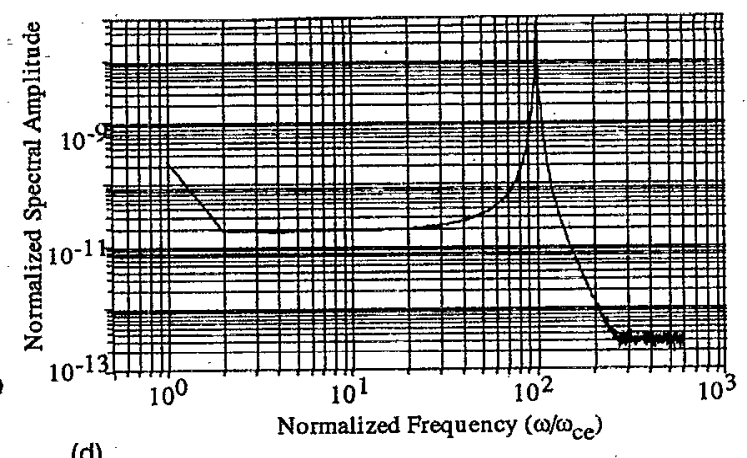

$\mathrm{X} / \mathrm{d}=0.0 ; \mathrm{Z} / \mathrm{d}=0.0$

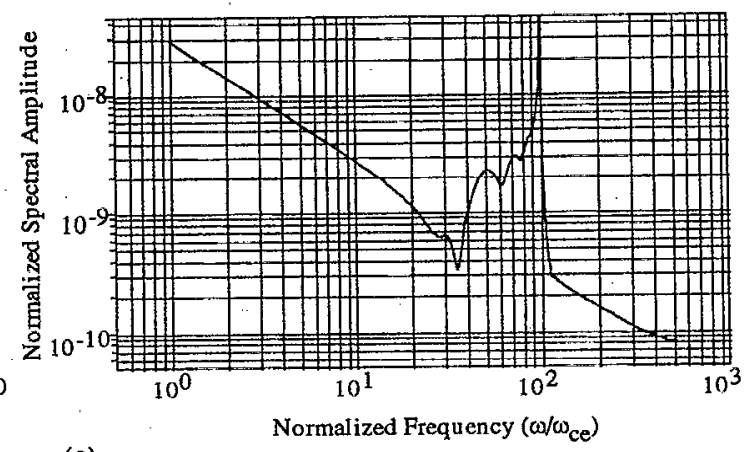

(e)

$\mathrm{X} / \mathrm{A}=0.0 ; \mathrm{Z} / \mathrm{A}=1.5$

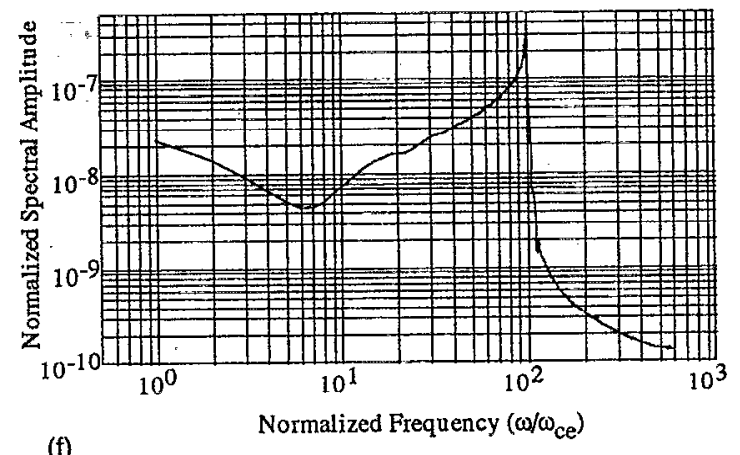

$\mathrm{X} / \mathrm{d}=0.0 ; \mathrm{Z} / \mathrm{d}=3.0$

(c)

Normalized Time $\left(f_{p e} \times t\right)$

$$
\mathrm{X} / \mathrm{d}=0.0 ; \mathrm{Z} / \mathrm{d}=3.0
$$

FIG. 7. Amplitude versus time and spectral amplitude versus frequency for the $y$ component of the electric field on plasina period time scales. (a)-(c) The electromagnetic solution for the $y$ component of the electric field normalized to $V_{0} B_{0} / c$ plotted as a function of time, normalized to the minimum cloud electron plasma period, at three locations in the plasma cloud; namely, $x=0, z=0$ (a); $x=0, z=1.5 d$ (b); $x=0, z=3.0 d$ (c). The corresponding spectral amplitude of the field for these three locations is plotted in (d)-(f), respectively, where frequency (plotted along the abscissa) is normalized to an electron cyclotron frequency and the Nyquist frequency was chosen to be six times the peak cloud electron plasma frequency.

center of the cloud and eventually out of the plasma. The dispersion relation for whistler waves is such that the group velocity $\left(V_{g}=2 c \sqrt{\omega \omega_{c c} / \omega_{p e}^{2}}\right)$ decreases with increasing density so that energy piles up as the pulse propagates toward the center of the cloud. In addition, whistler waves are dispersive with the high frequencies arriving first. The generation of these right-hand waves is not associated with any normal modes of the system but rather results from the self-consistent, large-scale evolution of a finite plasma and its corresponding electromagnetic radiation. This portion of the electromagnetic spectrum appears to be part of a broadband resonance about the ion cyclotron frequency as 


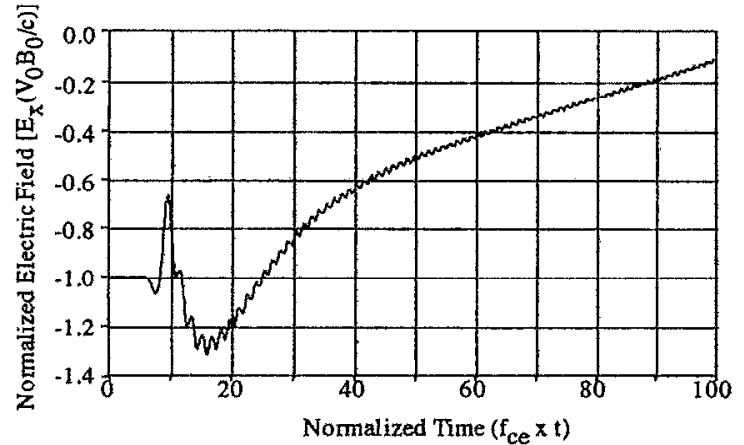

(a)

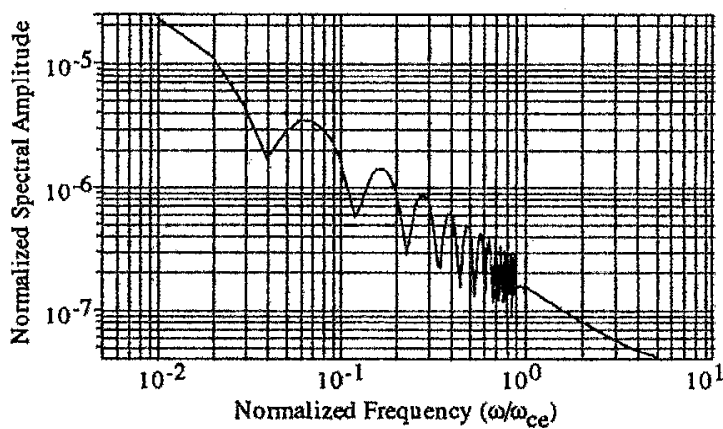

(d)
$\mathrm{XD}=0.0 ; \mathrm{ZNA}=0.0$

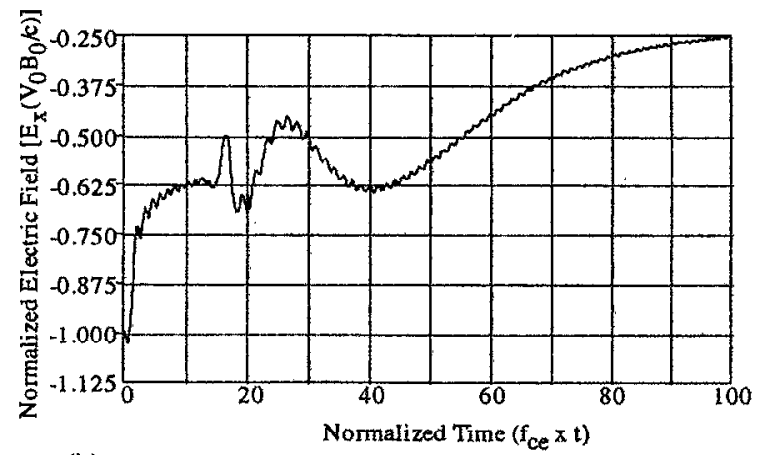

(b)

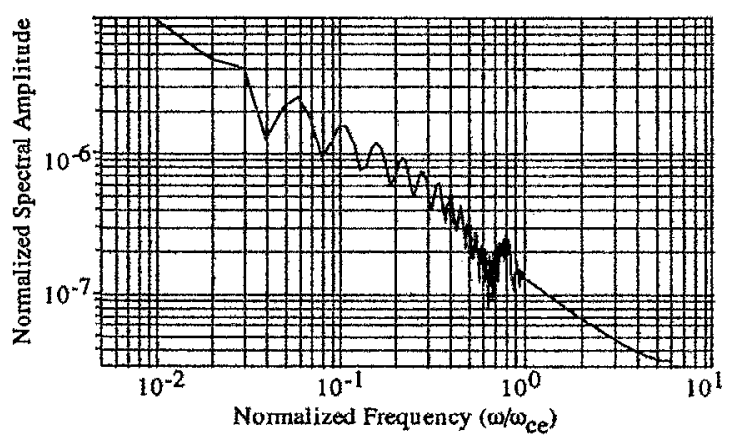

(e)
$X / D=0.0 ; Z / A=1.5$

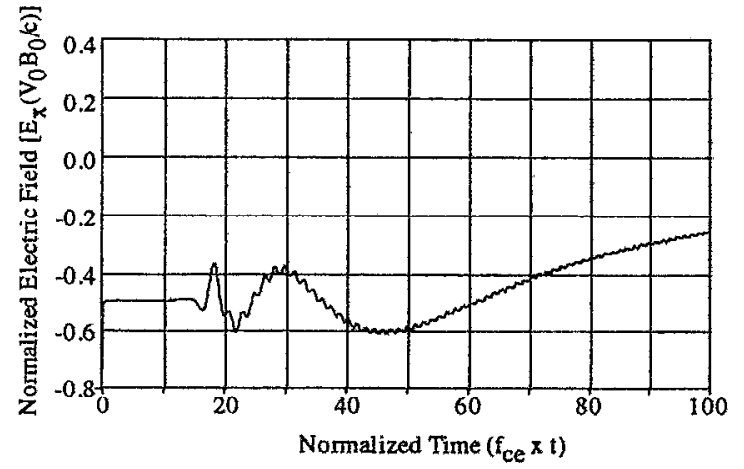

(c)

$\mathrm{X} D=0.0 ; \mathrm{Z}, \mathrm{A}=3.0$

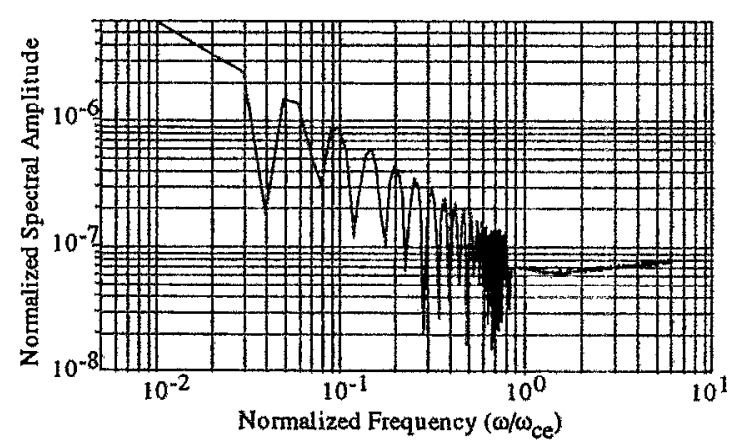

$(f)$

$X / D=0.0 ; Z A B=3.0$

FIG. 8. Amplitude versus time and spectral amplitude versus frequency for the $x$ component of the electric field on electron cyclotron time scales. (a)-(c) The electromagnetic solution for the $x$ component of the electric field normalized to $V_{0} B_{0} / c$ plotted as a function of time, normalized to the electron cyclotron period, at three locations in the plasma cloud; namely, $x=0, z=0$ (a); $x=0, z=1.5 d$ (b); $x=0, z=3.0 d$ (c). The corresponding spectral amplitude of the field for these three locations is plotted in (d)-(f), respectively, where frequency (plotted along the abscissa) is normalized to an electron cyclotron frequency and the Nyquist frequency was chosen to be six times the electron gyrofrequency.

will become evident in the following section that treats the evolution of the plasma cloud on ion cyclotron period time scales. The gradual decay of the polarization field indicates that a large part of the cloud momentum is being radiated away.

In the outer layers of the plasma, we see the superposition of two main pulses, one corresponding to the pulse observed at the center of the cloud propagating outward and the other which appears to have formed locally and which also propagates outward. The presence of the second pulse is evident from the number of lobes that appear in the spectral plot [Fig. 8(e)]. This second pulse is itself composed of two pulses separated in time by approximately eight electron cyclotron periods. A clearer picture of this process is obtained from the temporal sequence of spatial contours which will be presented in Sec. IV.

The $y$ component of the electric field exhibits similar properties except that the amplitude of the field increases dramatically in the outer layers. This effect is associated with the fact that the right-hand waves that carry positive 


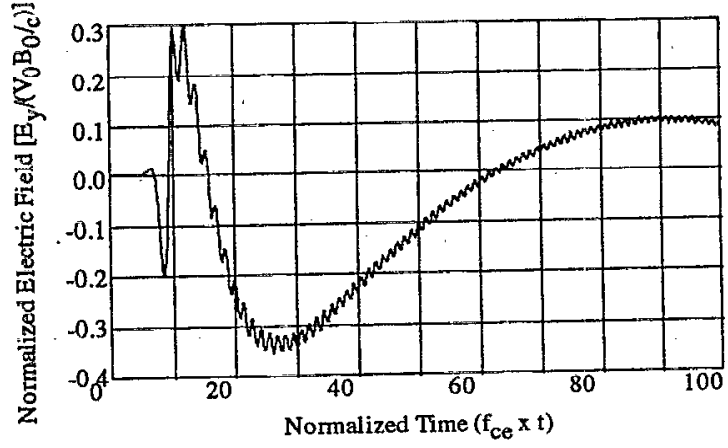

(a)

$\mathrm{XD}=0.0 ; \mathrm{ZKA}=0.0$

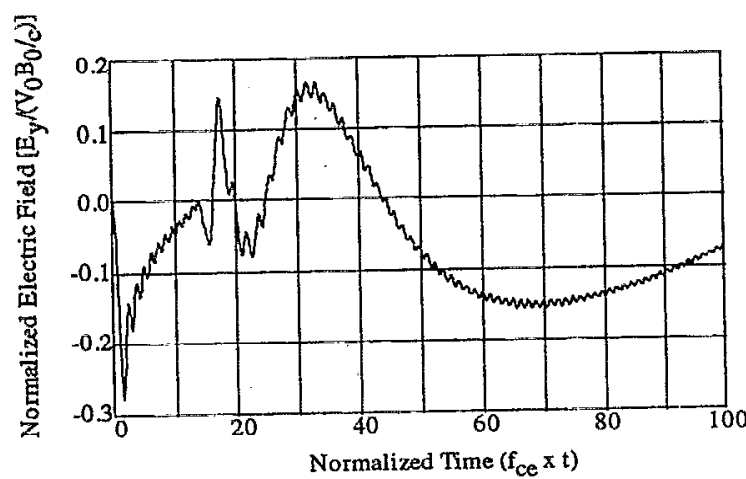

(b)

$\mathrm{X} / \mathrm{D}=0.0 ; \mathrm{Z} / \mathrm{d}=1.5$

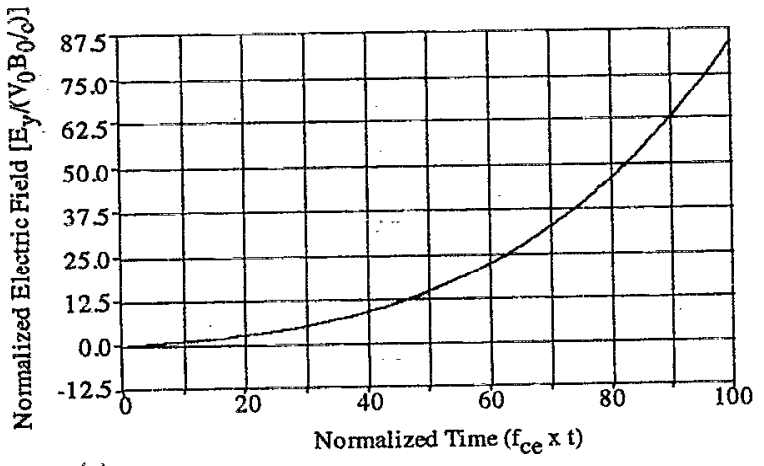

(c)

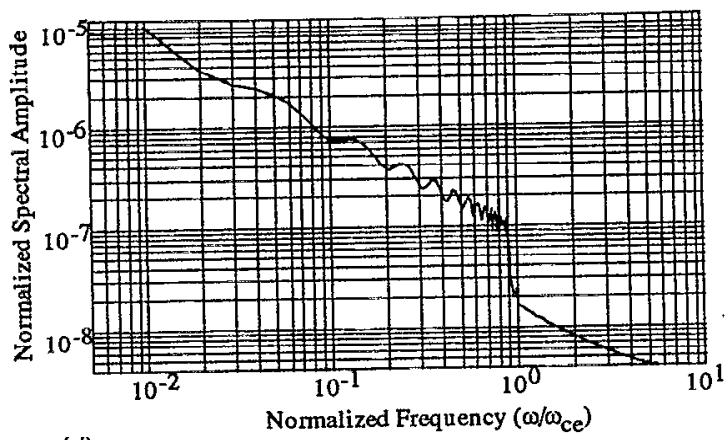

$X / D=0.0 ; Z / d=0.0$

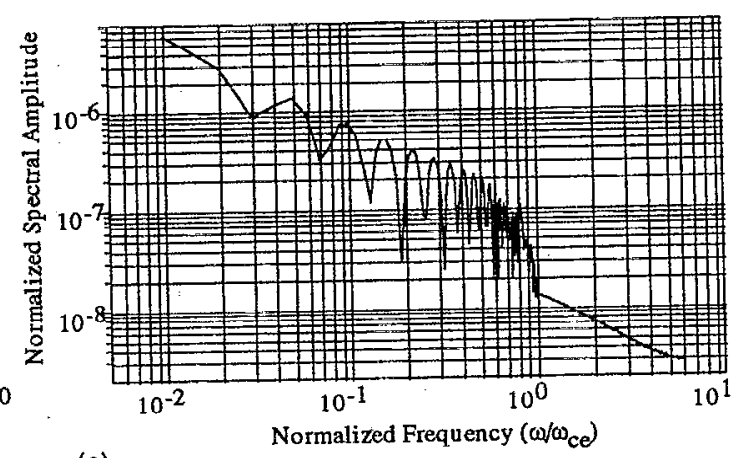

(e)

$\mathrm{XD}=0.0 ; \mathrm{Z} / \mathrm{d}=1.5$

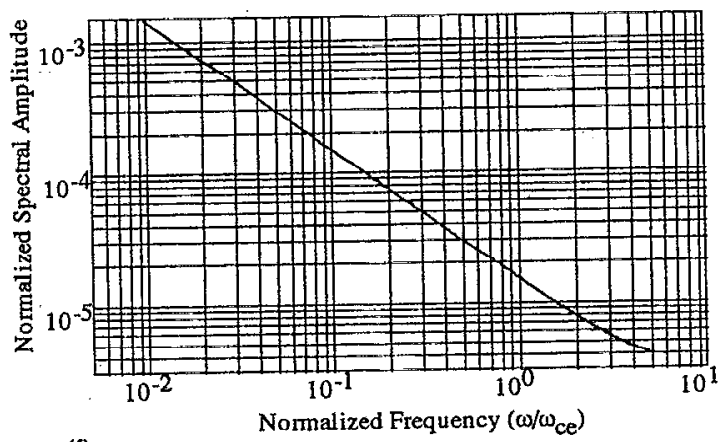

$\mathrm{X} / \mathrm{D}=0.0 ; \mathrm{Z} / \mathrm{d}=3.0$

FIG. 9. Amplitude versus time and spectral amplitude versus frequency for the $y$ component of the electric field on electron cyclotron time scales. (a) - (c) The electromagnetic solution for the $y$ component of the electric field normalized to $V_{0} B_{0} / c$ plotted as a function of time, normalized to the electron cyclotron period, at three locations in the plasma cloud; namely, $x=0, z=0$ (a); $x=0, z=1.5 d$ (b); $x=0, z=3.0 d$ (c). The corresponding spectral amplitude of the field for these three locations is plotted in (d)-(f), respectively, where frequency (plotted along the abscissa) is normalized to an electron cyclotron frequency and the Nyquist frequency was chosen to be six times the electron gyrofrequency.

$y$ momentum out of the center of the cloud become evanescent at low frequencies $\left(<2.8 \omega_{c i}\right)$ in the outer layers of the plasma and deposit their momentum in this region, resulting in a large $y$ electric field that transfers momentum to the ions. The momentum density carried by the righthand waves is a sizable fraction of the momentum density in the center of the cloud and is therefore much larger than the momentum density in the outer layers, hence the large amplitude of the $y$ electric field. We will elaborate further on this point in Sec. IV.

\section{Solution on ion cyclotron period time scales}

Results for the $x$ component of the electric field as a function of time, normalized to an ion cyclotron period, for 10 cycles are shown in Figs. 10(a)-10(c) with the corresponding Fourier transforms shown in Figs. 10(d)-10(f). A complementary set of plots for the $y$ component of the electric field is presented in Figs. 11(a)-11(f). With a Nyquist frequency of only six times the ion cyclotron period, we note that only the gross dynamics of the plasma cloud 


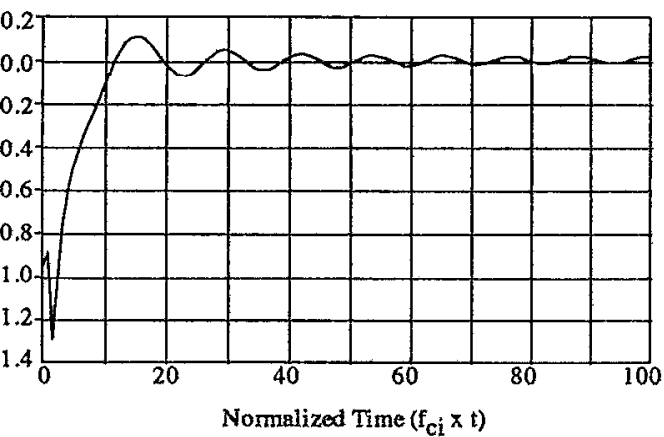

(a)

$X / D=0.0 ; Z / A=0.0$

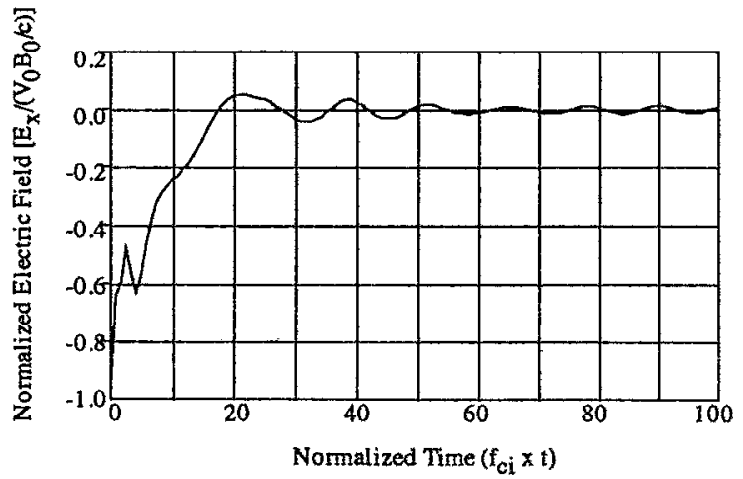

(b)

$\mathrm{X} D=0.0 ; \mathrm{ZA}=1.5$

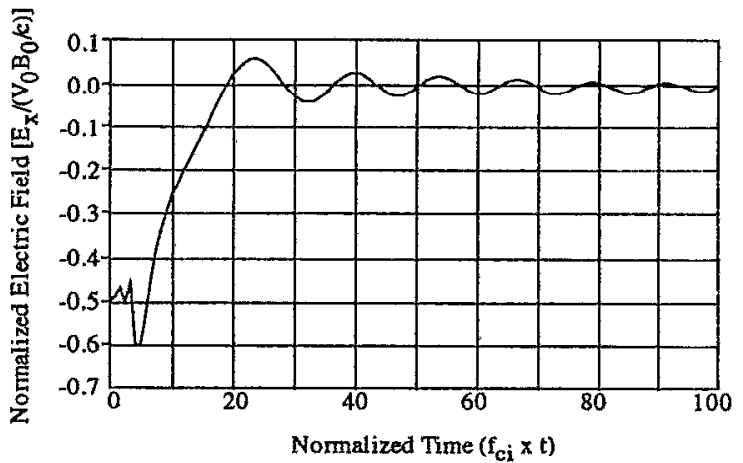

(c)

$\mathrm{XD}=0.0 ; \mathrm{ZC}=3.0$

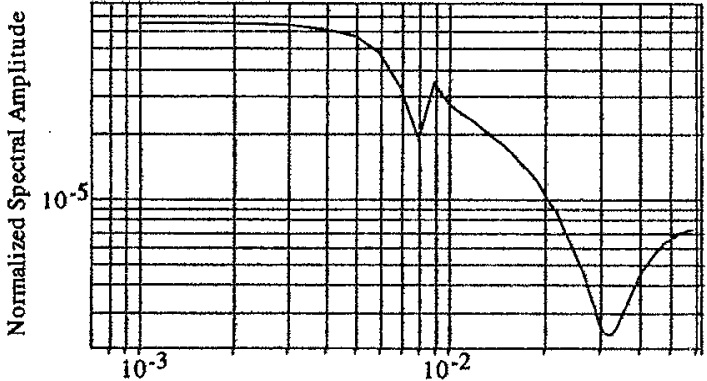

Frequency $\left(\omega / \omega_{\mathrm{ce}}\right)$

$\mathrm{X} D=0.0 ; \mathrm{ZAd}=0.0$

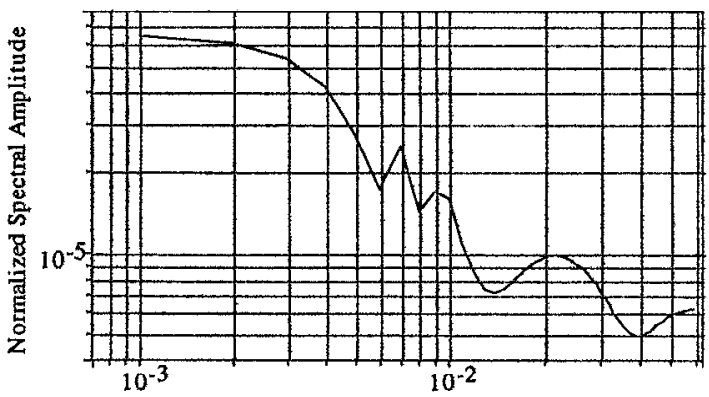

(e)
Frequency $\left(\omega / \omega_{c e}\right)$

$\mathrm{XD}=0.0 ; \mathrm{Z} / \mathrm{d}=1.5$

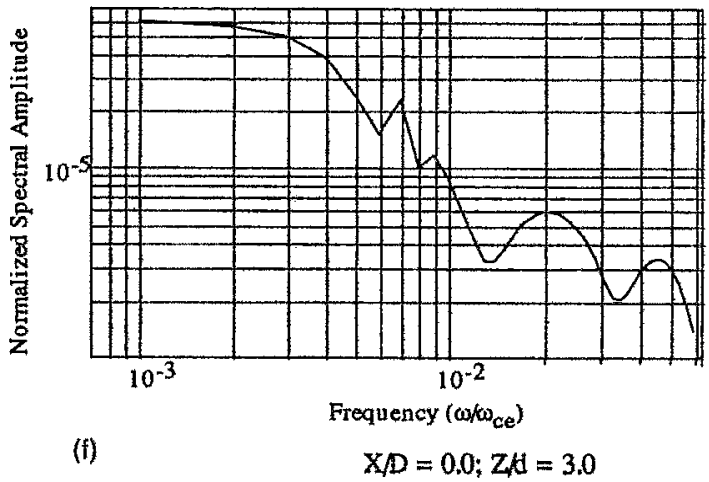

FIG. 10. Amplitude versus time and spectral amplitude versus frequency for the $x$ component of the electric field on ion cyclotron time scales. (a)-(c) The electromagnetic solution for the $x$ component of the electric field normalized to $V_{0} B_{0} / c$ plotted as a function of time, normalized to the ion cyclotron period, at three locations in the plasma cloud; namely, $x=0, z=0$ (a); $x=0, z=1.5 d$ (b); $x=0, z=3.0 d$ (c). The corresponding spectral amplitude of the field for these three locations is plotted in (d)-(f), respectively, where frequency (plotted along the abscissa) is normalized to an electron cyclotron frequency and the Nyquist frequency was chosen to be six times the ion gyrofrequency.

and radiation field will be resolved. Nonetheless, this time resolution is sufficient to describe the slowing down of the plasma cloud and the final equilibrium established between the plasma and the radiation field. In the center of the cloud, we see the rapid formation of the $x$ polarization field followed by the decay of the field to zero on a time scale comparable to an ion gyroperiod. The $x$ electric field then exhibits a damped oscillation about zero with a frequency near the ion gyroperiod. The spectral amplitude increases with decreasing frequency and has a peak at $0.9 \omega_{c i}$. The width of the spectrum extends into the lower-hybrid range with decreasing amplitude, consistent with radiative damping of ion gyromotion. The high-frequency component of the spectrum overlaps with the results obtained on the electron gyroperiod time scale and is consistent with those results. In the outer layers of the plasma, we see the superposition of two main pulses, one corresponding to the pulse observed at the center of the cloud propagating outward 


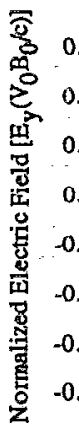

(a)

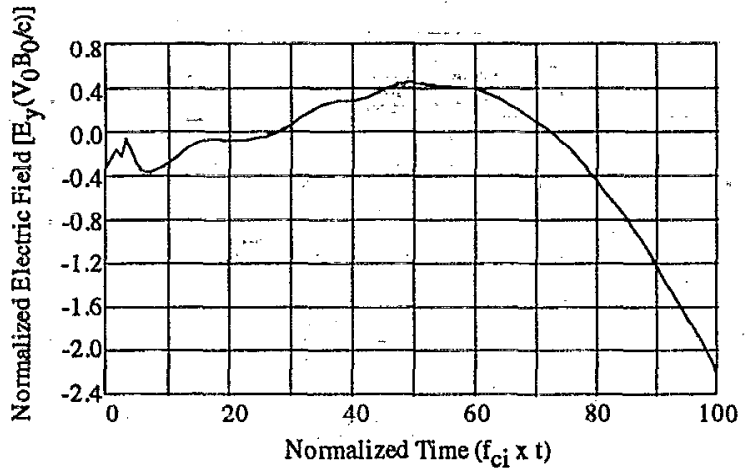

(b)

$\mathrm{XD}=0.0 ; \mathrm{Z} / \mathrm{d}=1.5$

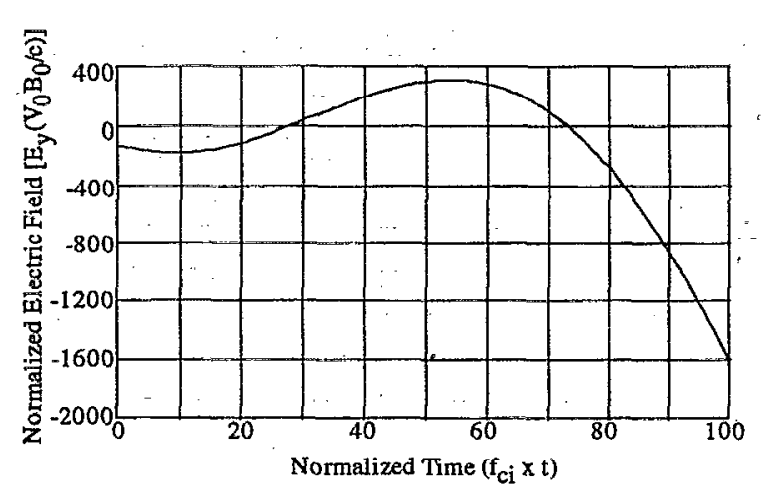

(c)

$\mathrm{X} / \mathrm{D}=0.0 ; \mathrm{Z} / \mathrm{d}=3.0$

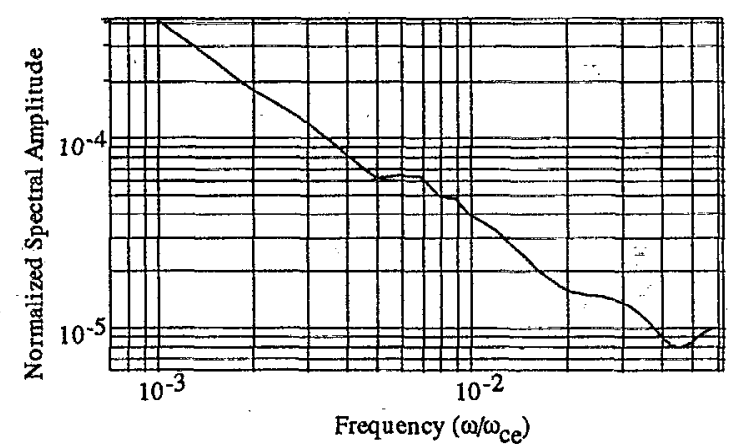

(e)

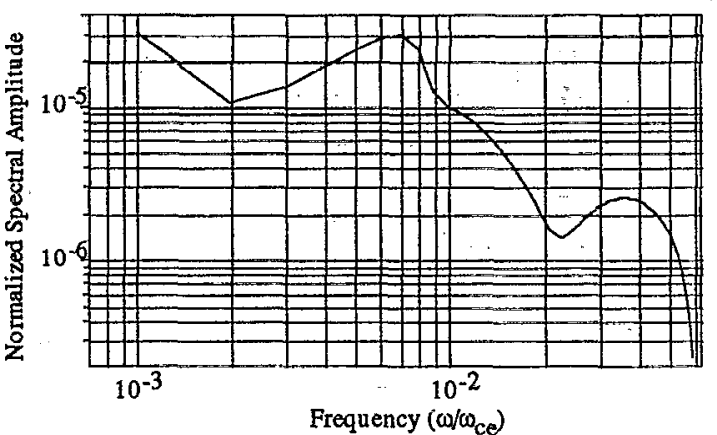

(d)

$\mathrm{XD}=0.0 ; \mathrm{Z} / \mathrm{d}=0.0$

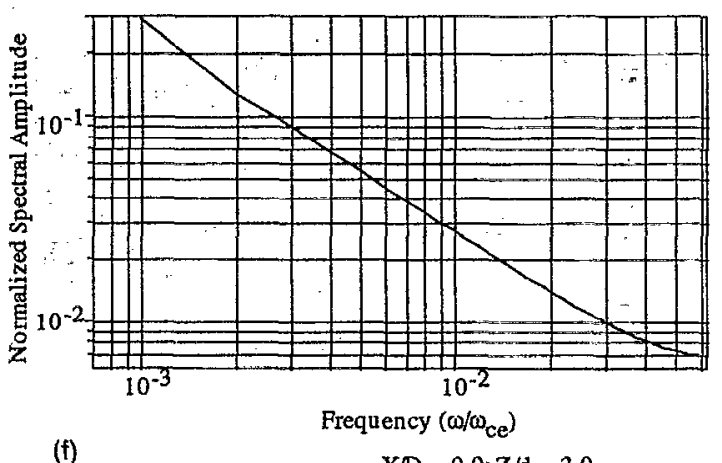

$\mathrm{XD}=0.0 ; \mathrm{Z} / \mathrm{d}=3.0$

FIG. 11. Amplitude versus time and spectral amplitude versus frequency for the $y$ component of the electric field on ion cyclotron time scales. (a)-(c) The electromagnetic solution for the $y$ component of the electric field normalized to $V_{0} B_{0} / c$ plotted as a function of time, normalized to the ion cyclotron period, at three locations in the plasma cloud; namely, $x=0, z=0$ (a); $x=0, z=1.5 d$ (b); $x=0, z=3.0 d$ (c). The corresponding spectral amplitude of the field for these three locations is plotted in (d)-(f), respectively, where frequency (plotted along the abscissa) is normalized to an electron cyclotron frequency and the Nyquist frequency was chosen to be six times the ion gyrofrequency.

and the other, which appears to have formed locally, possessing a spectral peak at approximately $0.7 \omega_{c i}$.

The $y$ component of the electric field exhibits somewhat different properties than the $x$ component. At the center of the cloud, the spectrum is shifted to lower frequencies with peaks occurring at $0.7 \omega_{c i}$ and $3.5 \omega_{c i}$. In the outer layers, we find a substantial increase in the field amplitude caused by continued momentum deposition in these regions as discussed in. subsection $\mathrm{C}$ of this section. We note that the ion cyclotron waves observed in the center of the cloud are left-hand polarized and therefore escape into vacuum.

\section{E. Partitioning of field and plasma momentum}

Momentum conservation under the approximations of this analysis is defined by Eq. (30) of Paper I. Integrating the various components of this equation over the plasma volume and over time and dividing by the spatially. inte- 

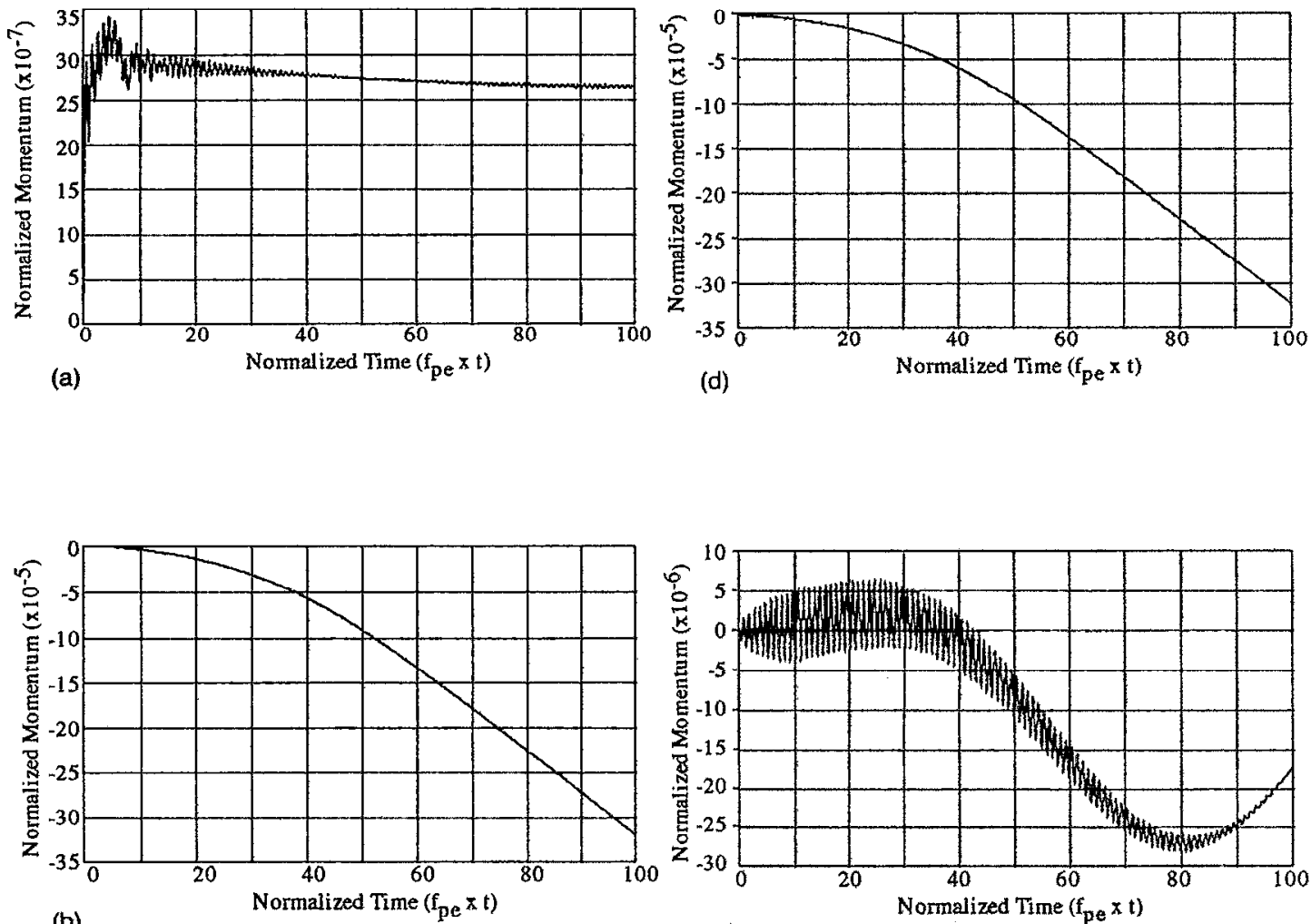

(e)
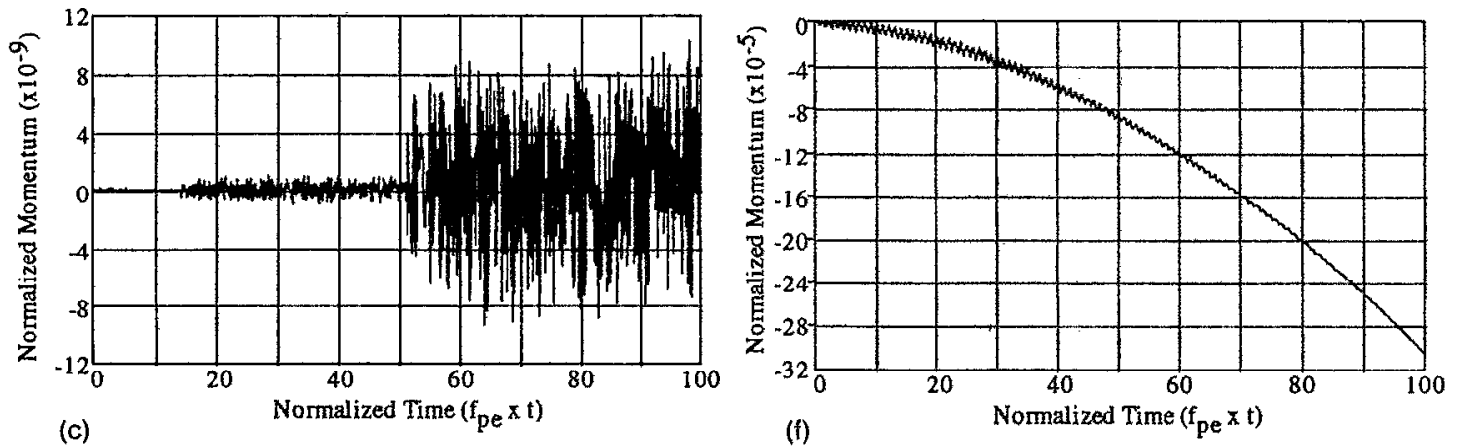

FIG. 12. Momentum conservation depicted on plasma period time scales. The integrated momentum stored in the electromagnetic fields (a), the total momentum lost through the $z$ surface of the plasma cloud by radiation (b), the total field momentum plus final plasma momentum minus initial plasma momentum (c), the integrated final plasma cloud momentum minus the initial plasma cloud momentum (d), the electron final integrated momentum minus initial electron momentum (e), and the ion final integrated momentum minus initial ion momentum all normalized to the total initial integrated cloud momentum are plotted as a function of time normalized to the minimum cloud plasma period for one electron cyclotron period.

grated initial momentum of the system (initially in the form of plasma momentum) yields a set of parameters for the final plasma cloud momentum minus the initial plasma cloud momentum, the total final momentum stored in the fields, and the net momentum carried away by the radiation field, all normalized to the initial plasma cloud momentum. These parameters are plotted as a function of time in Figs. 12(a)-12(f) for a Nyquist frequency equal to six times the maximum plasma frequency of the cloud, in Figs. 13(a)-13(f) for a Nyquist frequency equal to six times the electron gyrofrequency, and in Figs. 14(a)-14(f) for a Nyquist frequency equal to six times the ion gyrofrequency. First, we note that the total momentum stored in the electromagnetic field at any instant in time is always small compared to the total initial cloud momentum [see Figs. 12(a)-14(a)]. At early times, we see that only a small fraction $(\leqslant 0.0032)$ of the total cloud momentum is radiated away [see Figs. 12(b) and 12(d)]. On this time scale, the electron momentum changes by a maximum of $0.15 \%$ while the ion momentum changes by a maximum of 

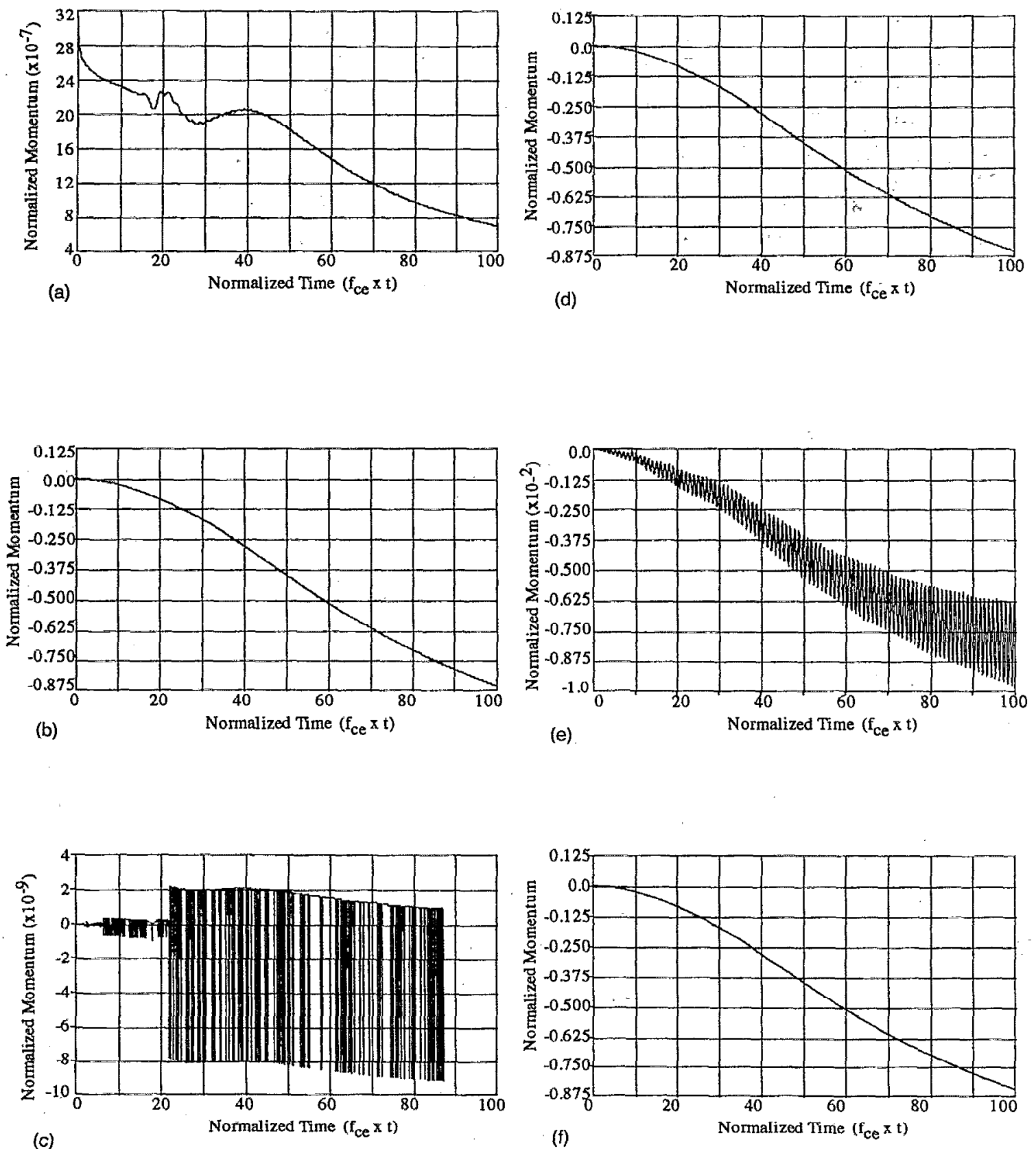

FIG. 13. Momentum conservation depicted on electron cyclotron period time scales. The integrated momentum stored in the electromagnetic fields (a), the total momentum lost through the $z$ surface of the plasma cloud by radiation (b), the total field momentum plus final plasma momentum minus initial plasina momentum (c), the integrated final plasma cloud momentum minus the initial plasma cloud momentum (d), the electron final integrated momentum minus initial electron momentum (e), and the ion final integrated momentum minus initial ion momentum all normalized to the total initial integrated cloud momentum are plotted as a function of time normalized to the electron cyclotron period for one ion cyclotron period.

$0.03 \%$ [Figs. 12(e) and 12(f)]. For time scales greater than an electron gyroperiod and out to one ion gyroperiod, we see that approximately $85 \%$ of the cloud momentum is radiated away [Figs. 13(b) and 13(d)]. On this time scale, both the electrons and ions lose approximately the same fraction of their total momentum [Figs. 13(e) and 13(f)]. For times greater than an ion gyroperiod, we find that the cloud settles into gyrotropic motion at a frequency somewhat less than an ion cyclotron frequency [Figs. 14(b) and 14(d)]. During this time, positive and negative momenta are being radiated away as the plasma cloud rotates.

Figures $12(\mathrm{c})-14(\mathrm{c})$ are plots of the total field plus plasma momentum as a function of time. We see that these plots are dominated by numerical noise about a value of zero indicating that momentum is conserved throughout our calculations. Because momentum in our analysis is linear in the field quantities, it is conserved independently to each order in the Hermite polynomial expansion. This will 

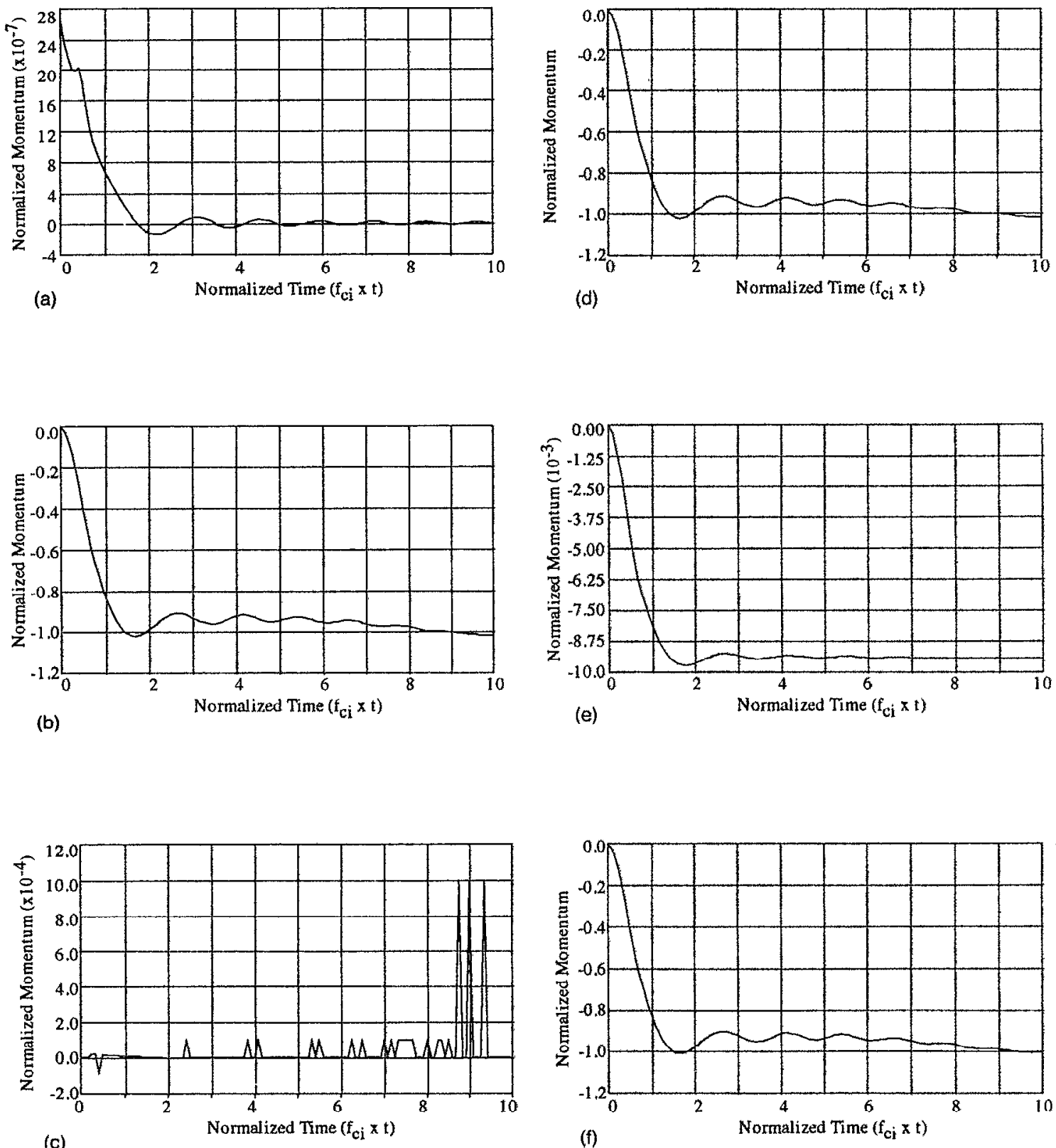

FIG. 14. Momentum conservation depicted on ion cyclotron period time scales. The integrated momentum stored in the electromagnetic fields (a), the total momentum lost through the $z$ surface of the plasma cloud by radiation (b), the total field momentum plus final plasma momentum minus initial plasma momentum (c), the integrated final plasma cloud momentum minus the initial plasma cloud momentum (d), the electron final integrated momentum minus initial electron momentum (e), and the ion final integrated momentum minus initial ion momentum all normalized to the total initial integrated cloud momentum are plotted as a function of time normalized to the ion cyclotron period for ten ion cyclotron periods.

not be the case for energy conservation as discussed below.

Finally, we note that the integrated momentum loss rate scales as

$$
\frac{1 d P_{\text {tot }}}{P_{\text {tot }} d t} \propto \frac{\omega_{c i}^{2}}{\omega_{p i}^{2}\left(1+m_{e} / m_{i}\right)} \frac{c^{2}}{d V_{\mathrm{ph}}} \frac{E_{x}}{V_{0} B_{0} / c},
$$

where $V_{\mathrm{ph}}$ is the phase velocity of the waves that carry momentum and energy out of the cloud and the density is evaluated at the center of the cloud. For times less than an ion gyroperiod, the wave spectrum is dominated by lower- hybrid waves with a group velocity $V_{\mathrm{ph}}=c \sqrt{\omega \omega_{c e} / \omega_{p e}^{2}}$ Taking $\omega=\omega_{c i}$ and substituting this expression into Eq. (27) yields

$$
\frac{1 d P_{\text {tot }}}{P_{\text {tot }} d t} \propto \frac{V_{\mathrm{A}}}{d} \frac{E_{x}}{V_{0} B_{0} / c}
$$

where $V_{\mathrm{A}}$ is the Alfven speed. With $E_{x} \approx V_{0} B_{0} / c$, the slowing-down time normalized to an ion gyroperiod scales as 


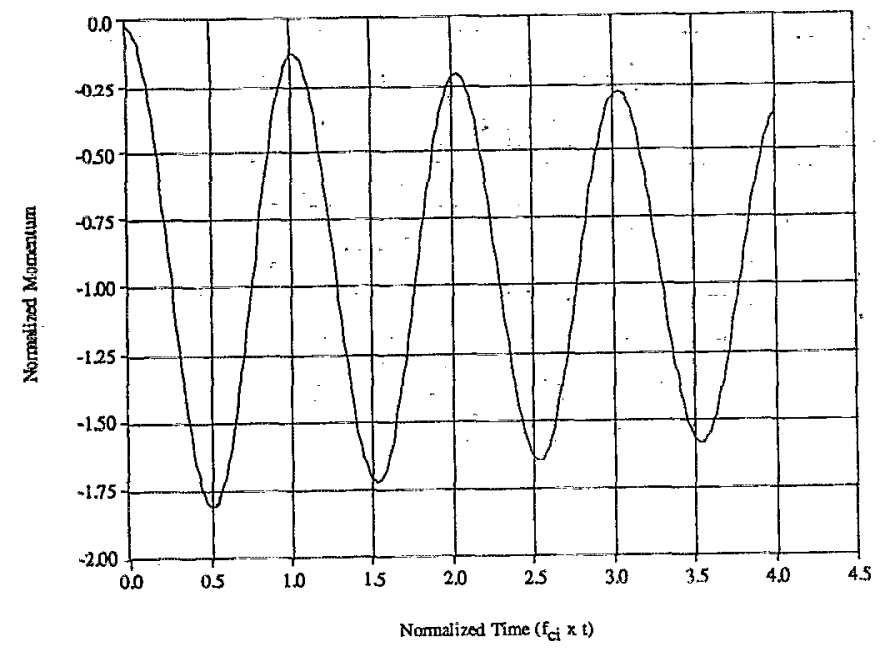

FIG. 15. The integrated final plasma cloud momentum minus the initial plasma cloud momentum, normalized to the total initial integrated cloud momentum plotted as a function of time normalized to the ion cyclotron period for four ion cyclotron periods and an ion-to-electron mass ratio of $2.5 \times 10^{5}$ (corresponding to the mass of a barium ion).

$$
f_{c i} \tau_{s} \propto \frac{d \omega_{p i}}{2 \pi c}
$$

With this scaling, it is possible for the slowing-down time to become much less than an ion gyroperiod, for example, with increasing ion mass. In reality, this is not a reasonable result because the ions must turn a sizable fraction of their gyro-orbit before the plasma momentum can be lost. We checked (29) numerically and found that, for small ion masses, the scaling held but that, at very large ion masses, the normalized slowing-down time saturated to 0.25 and that, in this limit, the plasma as a whole exhibited gyrotropic motion with an energy equal to half its initial energy. Numerical results illustrating this effect are shown in Fig. 15 for the parameters given in Table II but with an ion mass equal to that of the barium ion $\left(m_{i} / m_{p}=137\right)$. We interpret this result as follows. For slowing-down times longer than an ion gyroperiod, the ions are prevented from gyrating by the polarization field and radiation damping is dominated by lower-hybrid waves. Because the polarization field is maintained for times longer than an ion gyroperiod, momentum and energy (see discussion below) are exchanged between the plasma and the radiation field at approximately the same rate. As the braking time decreases below an ion gyroperiod, the polarization electric field exhibits a concomitant decrease and the ions begin to gyrate. The $y$ induction field causes the electrons to drift with the ions and no additional energy is stored in the polarization field. Note that this effect is not present in the electrostatic limit. In this regime, momentum is lost at a faster rate than energy. If the ions are permitted to continue to gyrate, their momentum is eventually lost on a time scale equal to $0.25 \bar{\tau}_{c i}$. Because the energy loss rate is slower, the plasma simply undergoes gyrotropic motion and radiates primarily ion cyclotron radiation until all of the energy is lost or background fields and/or plasma begin to influence the plasma cloud. In summary, the scaling law given by (29) predicts that the radiation damping time will increase as the ion mass increases but at a slower rate than the corresponding increase in the ion gyroperiod. As the normalized braking time decreases, the plasma begins to exhibit gyrotropic motion and all of the momentum is lost on a time scale equal to $0.25 \tau_{c i}$. In the latter regime, energy is radiated at a slower rate than momentum and most of the radiation appears as ion cyclotron waves.

\section{F. Partitioning of field and plasma energy}

Energy conservation under the approximations of this analysis is defined by Eq. (31) of Paper I. Integrating the various components of this equation over the volume of our calculations and over time and dividing by the spatially integrated initial energy of the system (initially in the form of plasma kinetic energy) yields a set of parameters for the final plasma cloud energy minus the initial plasma cloud energy, the total final energy stored in the fields, and the net energy carried away by the radiation field, all normalized to the initial plasma cloud energy. These parameters are plotted as a function of time in Figs. 16(a)-16(d) for a Nyquist frequency equal to six times the maximum plasma frequency of the cloud, in Figs. 17(a)-17(d) for a Nyquist frequency equal to six times the electron gyrofrequency, and in Figs. 18(a)-18(d) for a Nyquist frequency equal to six times the ion gyrofrequency. For times less than an electron gyroperiod, we find that the total plasma energy decreases by less than $0.1 \%$ [Fig. 16(d)] and that approximately $33 \%$ of this decrease is stored in the field [Fig. 16(a)] while the remaining $67 \%$ is radiated away [Fig. 16(b)]. On these time scales, we find that the zerothorder Hermite polynomial expansion will conserve energy in the system to an accuracy of $1 \%$. For times less than one ion gyroperiod we find that the total plasma cloud energy decreases by approximately $80 \%$ [Fig. 17(d)]. The field energy results begin to break down for times greater than 0.8 ion cyclotron periods [Figs. 17(a) $-17(\mathrm{c})$ ]. This effect results from the unusually large $y$ component electric field that develops in the outer layers of the plasma. The increase in amplitude of the $y$ electric field is caused by momentum deposition in these layers, as discussed previously, and shows up mathematically in our solution through the $y$ component of the eigenvectors. A corresponding increase in plasma kinetic energy also occurs in the outer layers of the plasma; however, the density weighting is such that these layers do not contribute significantly to the total cloud energy on an ion gyroperiod time scale. As noted in Paper I, the zeroth-order solution begins to break down in the outer layers because the $y$-component eigenvectors show strong spatial variations in these regions contrary to our initial assumptions. As a result, it will be necessary to include higher-order terms in our analysis in order to accurately model the low-density portion of the plasma cloud. Indeed, because the field energy terms are nonlinear, the departure from energy conservation is a good indication that higher-order terms are needed. We do not feel, however, that the overall quantitative conclusions of our analysis, as pertains to the bulk of the plasma motion, are 

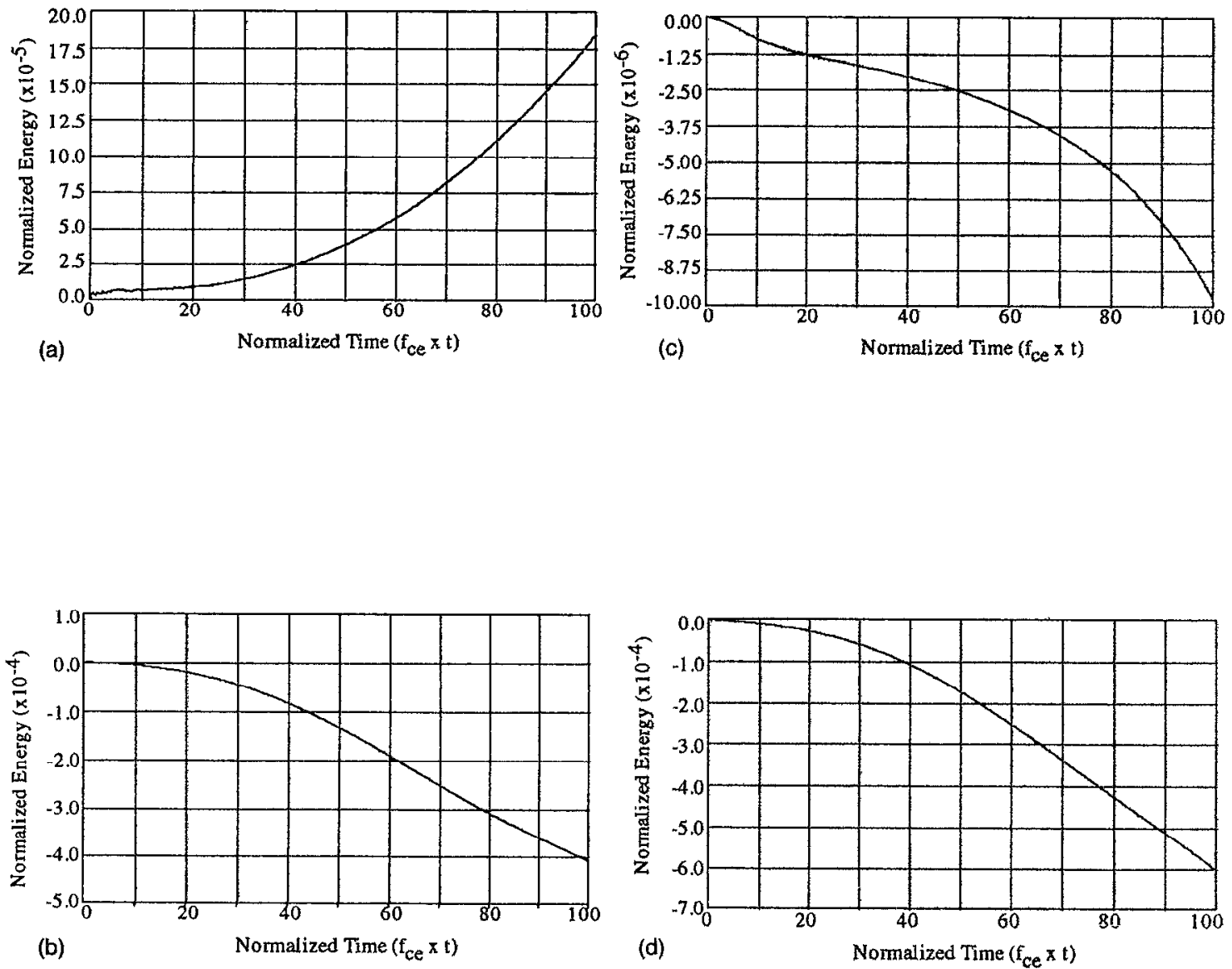

FIG. 16. Energy conservation depicted on plasma period time scales. The integrated kinetic energy stored in the electromagnetic fields (a), the total kinetic energy lost through the $z$ surface of the plasma cloud by radiation (b), the total field kinetic energy plus final plasma kinetic energy minus initial plasma kinetic energy (c), and the integrated final plasma cloud kinetic energy minus the initial plasma cloud kinetic energy (d), all normalized to the total initial integrated cloud kinetic energy are plotted as a function of time normalized to the minimum cloud plasma period for one electron cyclotron period.

affected for reasons that will be discussed below. Similar results are obtained for times greater than an ion gyroperiod [Figs. 18(a)-18(d)] as the $y$ electric field continues to increase in the outer layers.

The integrated energy loss rate scales as,

$$
\begin{aligned}
\frac{1 d E_{\mathrm{tot}}}{E_{\mathrm{tot}} d t} \propto \frac{\omega_{c i}^{2}}{\omega_{p i}^{2}\left(1+m_{\mathrm{e}} / m_{i}\right)} \frac{c^{2}}{d V_{\mathrm{ph}}}\left[\left(\frac{E_{x}}{V_{0} B_{0} / c}\right)^{2}\right. \\
\left.+\left(\frac{E_{y}}{V_{0} B_{0} / c}\right)^{2}\right]
\end{aligned}
$$

where all parameters were defined previously. This scaling is similar to that of the momentum loss rate except for the quadratic dependence on the field amplitudes. Substituting the phase velocity for lower-hybrid waves, we find

$$
\frac{1 d E_{\text {tot }}}{E_{\text {tot }} d t} \propto \frac{V_{\mathrm{A}}}{d}\left[\left(\frac{E_{x}}{V_{0} B_{0} / c}\right)^{2}+\left(\frac{E_{y}}{V_{0} B_{0} / c}\right)^{2}\right] .
$$

With $E_{x}$ and $E_{y}$ of order $V_{0} B_{0} / c$, the time scale for energy loss normalized to an ion gyroperiod scales as

$$
f_{c i} \tau_{s} \propto \frac{d \omega_{p i}}{2 \pi c} .
$$

This scaling is identical to that of the slowing-down time; however, as the amplitude of the "dc" polarization electric field decays, the energy loss rate will decrease faster than the momentum loss rate. Thus it is possible for the plasma cloud to settle into gyrotropic motion. If the momentum loss is rapid, i.e., $\tau_{s} \approx 0.25 \tau_{c i}$ as discussed above, the plasma will retain a substantial fraction of its energy for a number of ion gyroperiods (see, e.g., Fig. 15). For times larger than an ion cyclotron period, energy is radiated primarily as ion cyclotron waves.

\section{DISCUSSION}

\section{A. Comparison of electromagnetic and electrostatic results}

The electrostatic solution at high densities for the polarization electric field $\left(E_{x}\right)$ consists of a high-frequency oscillation at the upper-hybrid frequency with amplitude $V_{0} B_{0} / c$ about a mean value of $-V_{0} B_{0} / c$. A similar result is obtained for the electromagnetic solution in the central portion of the plasma cloud at early times except that the oscillation frequency is shifted slightly and two modes are present as a result of the generation of an induction field in 


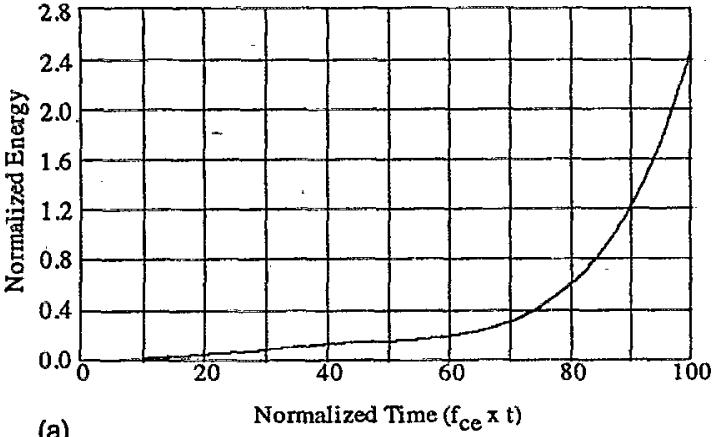

(a)

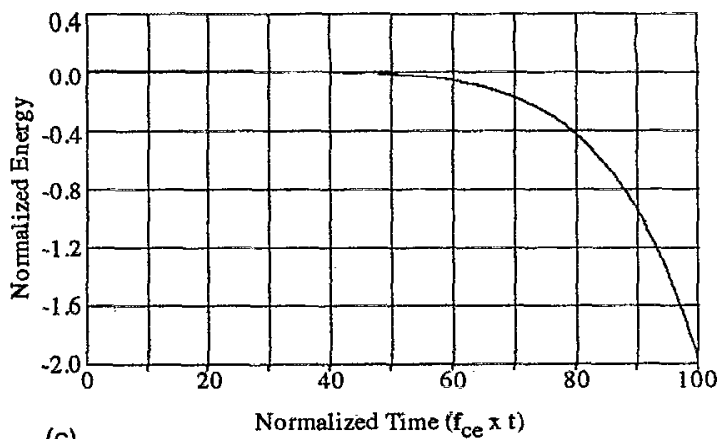

(c)

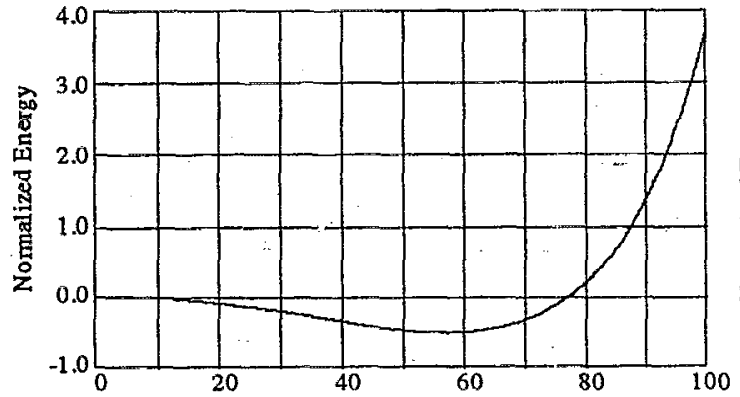

(b)

Normalized Time $\left(f_{c e} \times t\right)$

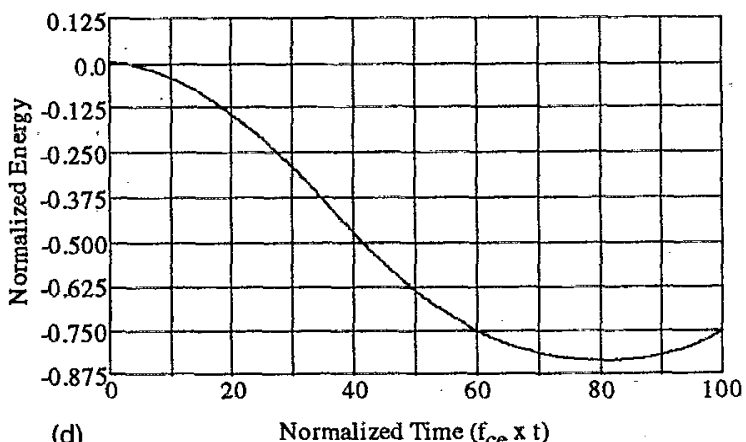

(d)

Normalized Time $\left(f_{c e} \times t\right)$

FIG. 17. Energy conservation depicted on electron cyclotron pcriod time scales. The integratcd kinetic cncrgy stored in the clectromagnetic ficlds (a), the total kinetic energy lost through the $z$ surface of the plasma cloud by radiation (b), the total field kinetic energy plus final plasma kinetic energy minus initial plasma kinetic energy (c), and the integrated final plasma cloud kinetic energy minus the initial plasma cloud kinetic energy (d), all normalized to the total initial integrated cloud kinetic energy are plotted as a function of time normalized to the electron cyclotron period for one ion cyclotron period.

the $y$ direction. As the plasma radiates energy and momentum out of the central portion of the cloud, the amplitude of the oscillations decreases and a transition to lowerhybrid whistler waves occurs. Low-amplitude lower-hybrid oscillations are also present at high densities in the electrostatic solution [see Eq. (12)] and it is natural to assume that, as the amplitude of the high-frequency oscillations decreases, a transition to low-frequency oscillations will occur. As the plasma density decreases, the electrostatic solution shows a transition from upper-hybrid oscillations to electron cyclotron oscillations, to lower-hybrid oscillations, and finally to ion cyclotron oscillations [see Fig. 1(b)]. A similar effect is obtained in the electromagnetic solution except that local oscillations are generally masked by radiation propagating through various regions from different portions of the plasma cloud. At early times, highfrequency radiation from the central portion of the plasma cloud tends to dominate the spectrum of field oscillations in the outer layers. As time progresses, the lower-frequency waves produced primarily in the outer layers result in the formation of several whistler pulses that propagate through the interior of the plasma cloud and that lead ultimately to the slowing down of the plasma in these regions. The for- mation of these pulses and their propagation through the center of the cloud can be seen in the temporal sequence of two-dimensional contour plots shown in Figs. 19(a)19(f). After 40 electron cyclotron periods [Figs. 19(g)19(1)], the lower-hybrid waves emerge from the center of the cloud as two large pulses that carry a substantial fraction of the initial momentum and energy of the cloud into vacuum. At late times, times greater than an ion cyclotron period, the plasma relaxes into gyrotropic motion at frequencies somewhat less than the ion gyrofrequency. By this time, most of the plasma energy and momentum has been radiated away.

At high densities, the partitioning of energy and momentum in the electrostatic limit is such that an equal but small amount of plasma momentum and energy is stored in the fields at any instant in time (see Fig. 3). For the parameters used in our calculations, this partitioning will apply throughout most of the plasma cloud. The electromagnetic results indicate that the cloud radiates away both its energy and momentum on the same time scale, in agreement with the partitioning obtained in the electrostatic results. As noted previously, the time scale for momentum loss scales as $\tau_{s} \propto d / V_{\mathrm{A}}$. This slowing-down time also ap- 

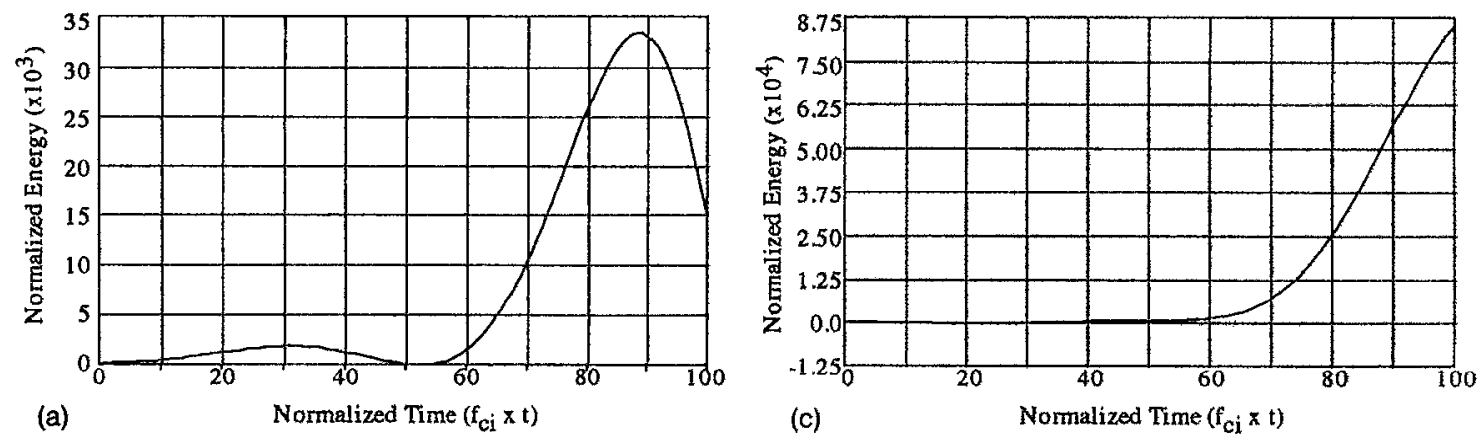

(c)

Normalized Time $\left(f_{\mathrm{ci}} \times t\right)$
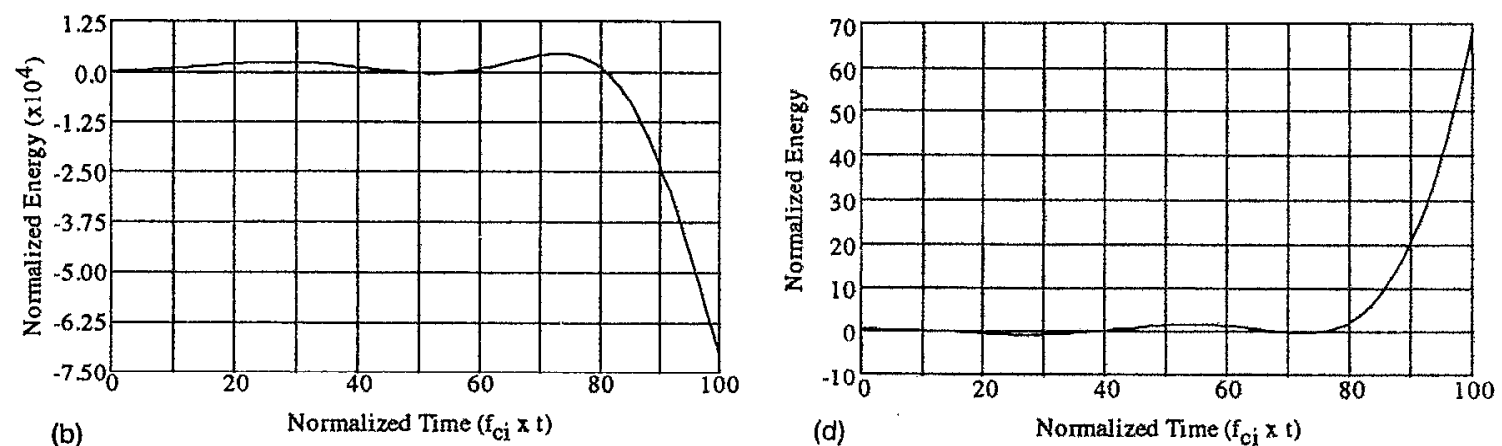

FIG. 18. Energy conservation depicted on ion cyclotron period time scales. The integrated kinetic energy stored in the electromagnetic fields (a), the total kinetic energy lost through the $z$ surface of the plasma cloud by radiation (b), the total field kinetic energy plus final plasma kinetic energy minus initial plasma kinetic energy (c), and the integrated final plasma cloud kinetic energy minus the initial plasma cloud kinetic energy (d), all normalized to the total initial integrated cloud kinetic energy are plotted as a function of time normalized to the ion cyclotron period for ten ion cyclotron periods.

plics to the time for energy loss for high-density plasma clouds. In the previous section, we noted that the momentum loss time scale cannot be less than a quarter of an ion gyroperiod and that, in this regime, the energy loss rate decreases significantly. The latter effect is due, in part, to the fact that the $y$ induction field allows the plasma, as a whole, to drift in the $x$ direction thereby reducing the amount of energy stored in the fields and eventually radiated. The $y$ induction field does not exist in the electrostatic calculations. As a result, for time scales greater than an electron gyroperiod the electrons become tied to the field lines and the ion motion across the field stores more energy in the $x$ polarization field than would occur in the electromagnetic case.

\section{B. Limitations of the zeroth-order solution}

As noted in Paper I, the approximations of geometrical optics are invalidated when the vacuum wavelength and dielectric wavelength of the waves become comparable to the dimensions of the plasma cloud. In this regime, it is necessary at a minimum to allow for spreading of electromagnetic waves such that the wave vectors have a sizable component in the $x$ direction and thus to include higherorder terms in the Hermite polynomial expansion. Our zeroth-order solution enters this regime at low frequencics and low densities and our results can be seen to break down in the outer layers of the plasma at times approaching an ion gyroperiod. This effect is directly manifested in the $y$ component of the eigenvectors, as discussed in Paper $I$, in the magnitude of the $y$ component of the electric field, and in the energy conservation equations as discussed above. We believe, however, that the basic conclusions of this paper regarding the radiation damping time, the spectrum of waves emitted, and the details of the plasma evolution in the interior of the cloud will remain unaffected for the following reasons. First, the breakdown of the solution is limited to the outer layers of the plasma and to late times ( $>$ ion gyroperiod). Second, the $x$ component of the electric field that effectively defines the $y$ momentum carried by the radiation field $\left(P_{y}=E_{x} \times B_{0} / 4 \pi c\right)$ is well behaved throughout the plasma cloud and for all time. This result also accounts for the fact that momentum is conserved throughout and, when coupled with the electrostatic prediction for energy and momentum partitioning, it lends credence to the calculated energy decay rate. Third, while the integrated field energy becomes unrealistic on time scales of order the ion gyroperiod, the total plasma kinetic energy remains well behaved out to seven ion cyclotron periods, well beyond the slowing-down time [see Fig. 17(c)]. This result is indicative of the fact that the break- 


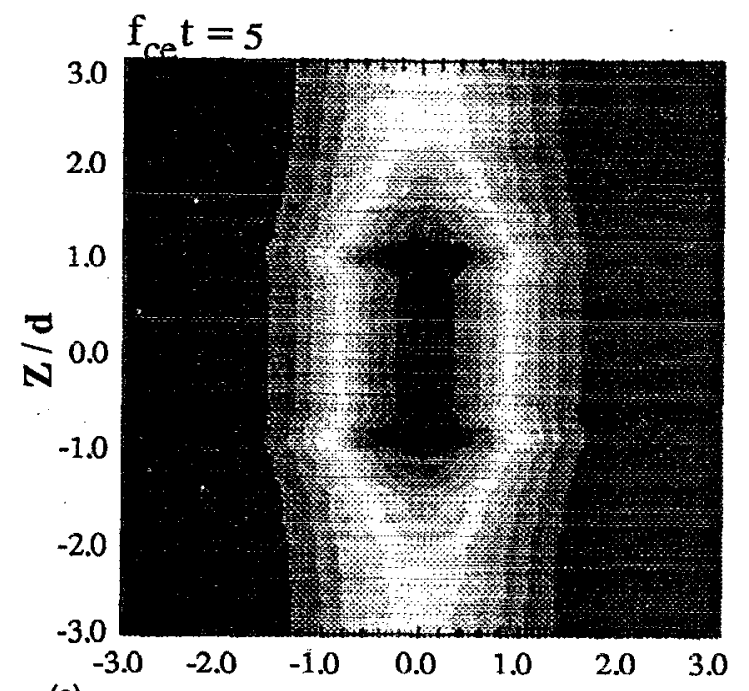

(a)
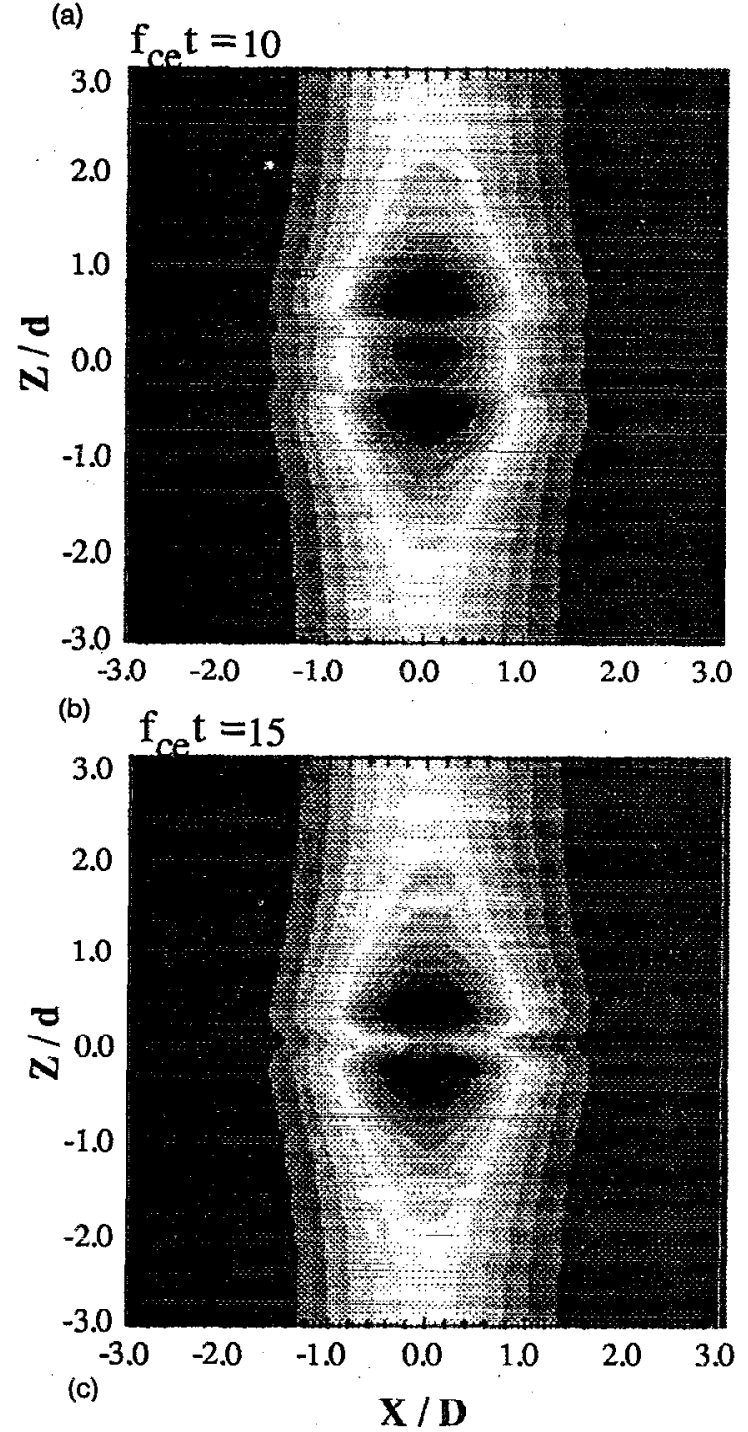

RELATIVE
AMPLITUDE

$-0.608 \mathrm{E}-04$

$-0.808 \mathrm{E}-01$

$-0.182 E+00$

$-0.272 \mathrm{E}+00$

$-0.383 E+00$

$-0.454 \mathrm{E}+00$

$-0.545 E+00$

$-0.635 \mathrm{E}+00$

$-0.726 \mathrm{E}+00$

$-0.817 \mathrm{E}+00$

$-0.907 \mathrm{E}+00$

$-0.998 E+00$

$-0.108 \mathrm{E}+01$

$-0.118 \mathrm{E}+01$

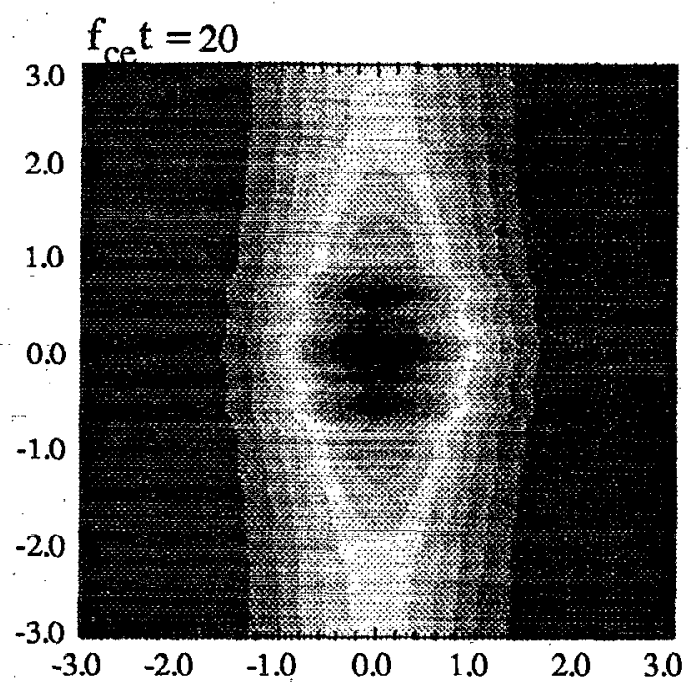

(d)

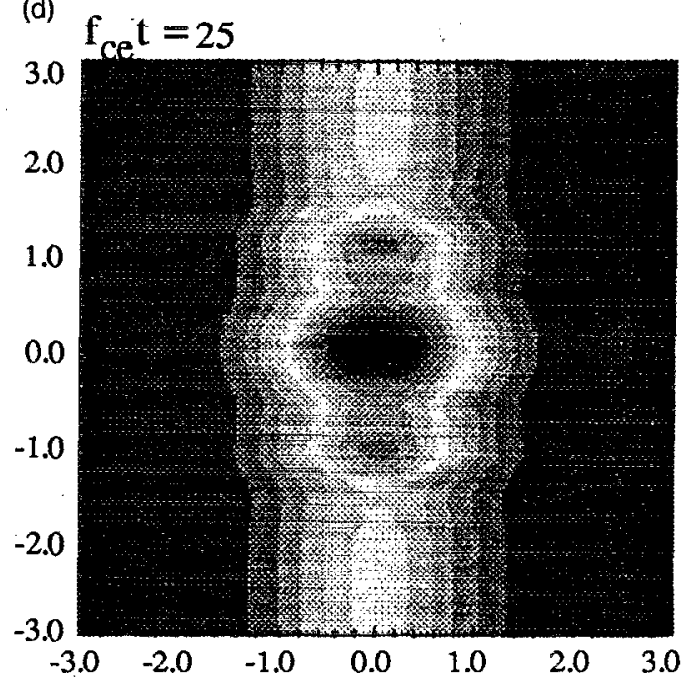

(e)

(e) $f_{c e} t=30$

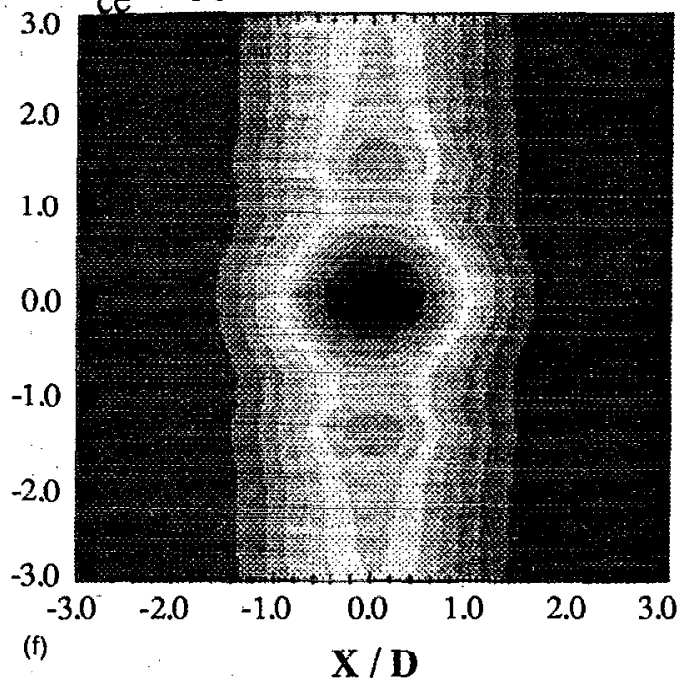

FIG. 19. Temporal sequence of two-dimensional contour plots for the $x$ component of the electric field. In these figures, the cloud motion is out of the page while the magnetic field points up the page along the $z$ direction. Snapshots of the electric field strength at intervals of five electron cyclotron periods out to 30 periods are shown in (a)-(f). (g)-(1) show the evolution of the electric field out to 125 electron cyclotron periods. The formation of lower-hybrid pulses and their propagation through the center of the cloud are clearly seen in these figures. 

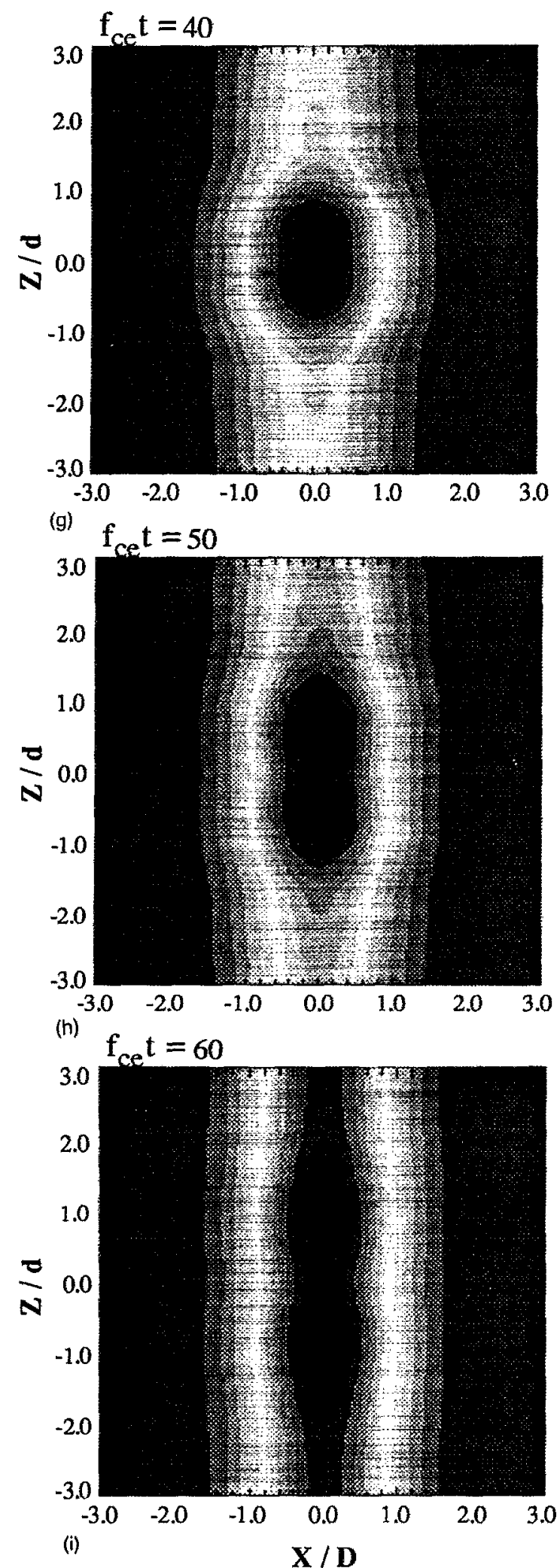

\section{RELATIVE \\ AMPLITUDE}

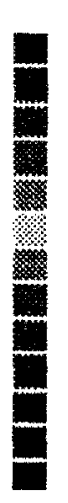

$-0.583 E-04$ $-0.468 \mathrm{E}-01$

$-0.918 \mathrm{E}-01$

$-0.138 \mathrm{E}+00$

$-0.184 E+00$

$-0.228 \mathrm{E}+00$

$-0.270 \mathrm{E}+00$

$-0.331 \mathrm{E}+00$

$-0.387 \mathrm{E}+00$

$-0.413 \mathrm{E}+00$

$-0.459 E+00$

$-0.505 E+00-2.0$

$-0.550 \mathrm{E}+00$ $-0.592 \mathrm{E}+00$

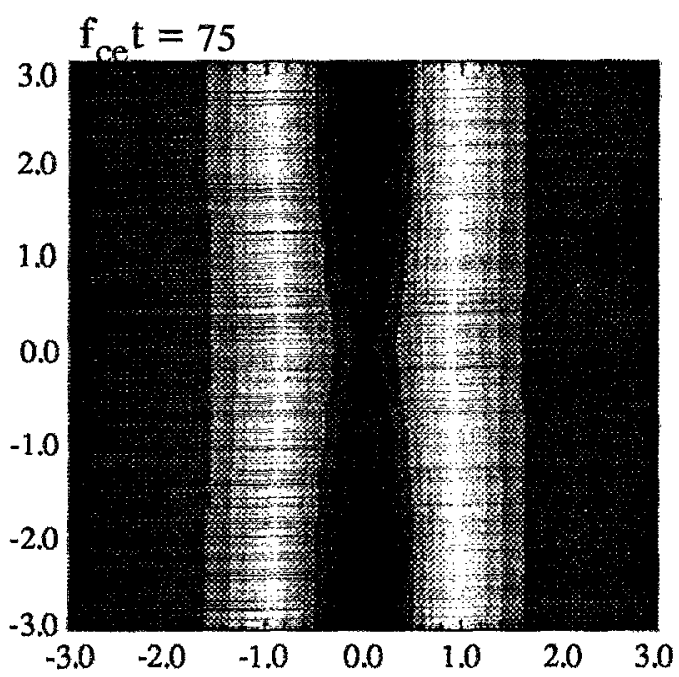

(j)

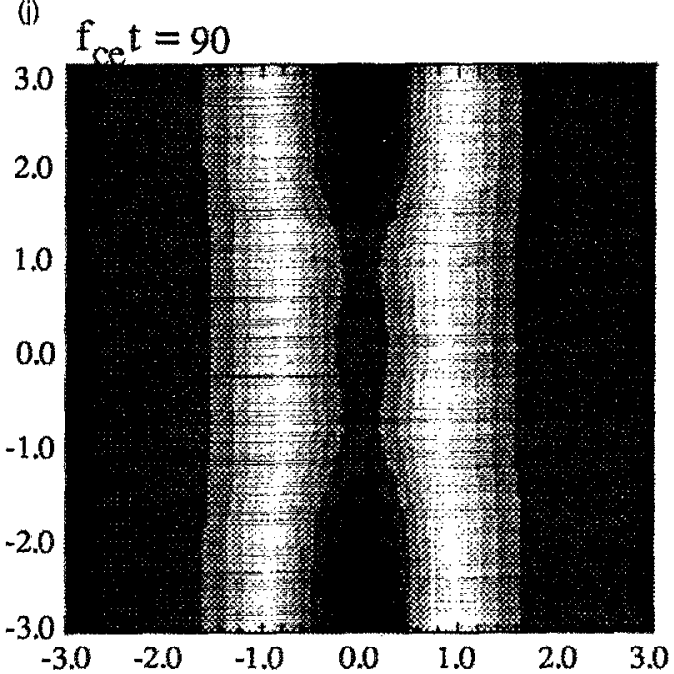

(k) $f_{\mathrm{ce}} t=125$

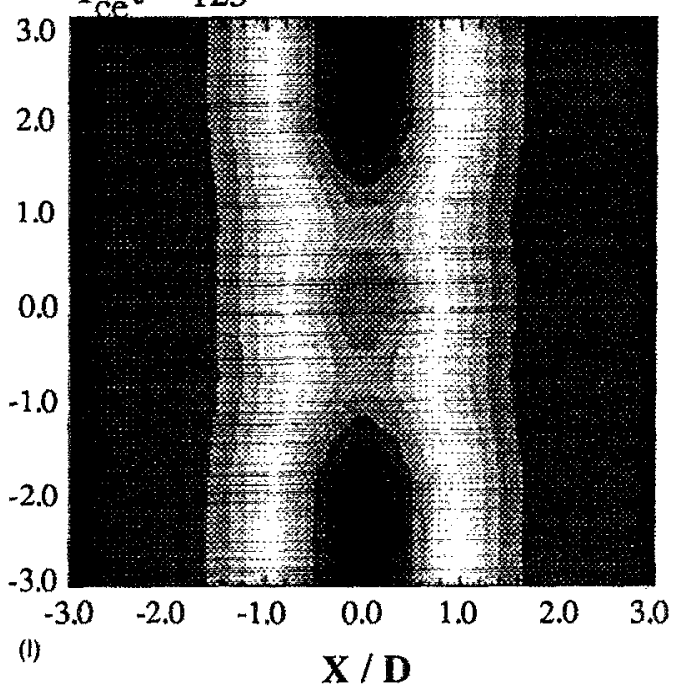

FIG. 19. (Continued.) 
down of the zeroth-order solution is isolated to the outer layers of the plasma. Finally, we note that large bipolar electric fields parallel to the beam direction (our $y$ direction) with magnitude several times $V_{0} B_{0} / c$ have been measured in recent critical velocity experiments ${ }^{5,6}$ along with the arrival of an anomalous population of energetic ions and electrons. ${ }^{5}$ While we cannot make a quantitative comparison with these measurements, the large $y$-component fields obtained in our calculations are in qualitative agreement with observation. Recall that these anomalously large fields originate from a deposition of wave momentum carried from the center of the cloud to the outer layers. In order to quantify these effects, however, it will be necessary to include higher-order terms in the Hermite polynomial expansion. We note that the addition of a background plasma will not only allow us to model ionospheric releases but will have the effect of decreasing the dielectric wavelength of the low-frequency waves thereby improving the accuracy of the zeroth-order term in the outer layers of the plasma cloud.

Finally, because the spectral plots shown in Figs. 6-11 are obtained in essence for finite-time windows, it is possible for both the low-frequency and high-frequency information in any single plot to be distorted. The former because of the low-frequency resolution afforded by the finite window size and the latter because of the abrupt cutoff of the time window. These effects, however, are severely mitigated in our analysis because of the method used to take the inverse Laplace transform. In all cases, the accuracy of our results was checked by increasing the number of aliasing terms and performing our calculations for a time window four times the size shown in the plots as prescribed by Dubner and Abate.?

\section{Observables}

The most obvious observable predicted by our analysis is the rapid braking of the plasma cloud. An estimate of the slowing-down time can be readily obtained from optical images of chemical releases as a function of time. Using the scaling described in Secs. III E and III F, a direct comparison between theory and experiment is possible, provided detailed geometrical effects are not important. In the absence of a background plasma and at high plasma densities, our analysis shows that the cloud momentum is carried away primarily by lower-hybrid whistler and ion cyclotron waves. The radiation spectrum is such that a highfrequency (near the peak plasma frequency of the cloud), chirped pulse with the higher frequencies arriving first is emitted initially, followed by lower-hybrid whistler pulses, and finally by ion cyclotron waves just below the ion cyclotron frequency. On time scales longer than an ion gyroperiod, our analysis predicts that the plasma cloud would exhibit gyrotropic motion. In addition, we expect to see the formation of a strong $y$ electric field and the presence of energetic ions in the outer layers of the cloud.

Many of the effects described above have been observed in recent barium chemical release experiments. The rapid braking of the plasma cloud was observed in both magnetospheric and ionospheric releases performed as part of the CRRES experiments ${ }^{11}$ and in the barium CRIT II release. ${ }^{6}$ Intense electromagnetic radiation at frequencies above the barium ion gyrofrequency (lower-hybrid range) and in the vicinity of the ion gyrofrequency were measured a few kilometers down field from the release in both CRRES and CRIT II experiments. ${ }^{3,6}$ Downstream electric field measurements in the CRIT I experiment ${ }^{5}$ indicated the presence of a burst of high-frequency ( $>9 \mathrm{kHz}$ ) "precursor waves" and bursts of waves at frequencies above the ion gyrofrequency. Very intense low-frequency (20-70 $\mathrm{Hz}$ ) magnetic perturbations with unresolved highfrequency oscillations were also measured in the CRIT I experiment. ${ }^{5}$ Downstream electric field measurements in the Condor critical velocity experiment ${ }^{4}$ revealed the existence of oxygen lower-hybrid and barium lower-hybrid waves. In the CRIT II experiment, ${ }^{6}$ bursts of "Alfvenic" radiation separated in time by a barium ion gyroperiod were measured, indicating that the plasma cloud was exhibiting gyrotropic motion. While these observations, on the whole, are in qualitative agreement with the results expected from our analysis, a more quantitative comparison will have to be performed in order assess the role played by radiation damping in these chemical releases.

\section{Quantitative predictions}

Given the results obtained in this paper, it is possible to make some quantitative predictions for low-beta magnetospheric releases. We note that the parameters given in $\mathrm{Ta}$ bles I and II yield a nominal value for the dynamic beta approximately equal to 2 . However, the perturbation magnetic field obtained in our solutions is found to be small compared to the ambient field throughout the calculation except in the outer regions of the plasma at late times. Thus our solution is at least self-consistent over the important temporal and spatial ranges of interest. In general, some fraction of the plasma kinetic energy will be expended in distorting the ambient magnetic field, producing a configuration similar to that observed in the Ampte comet releases. However, this effect is expected to be small simply because the dynamic beta is not large enough in the cross-field geometry associated with these releases to permit a strong-enough current to develop. Instead, a strong polarization field will form and this is precisely the scenario treated in our analysis.

Using the release parameters in Table I, the simulation parameters in Table II, and the scaling law for momentum loss (29) with allowance for the saturation of the slowing down time, we obtain a normalized braking time $f_{c i} \tau_{s}=4.2$ and $\tau_{s}=4.7 \mathrm{sec}$ (where $\tau_{c i}=1.12 \mathrm{sec}$ ). The peak amplitude of the high-frequency waves will equal approximately $E_{\mathrm{hf}} \approx V_{0} B_{0} / c=54 \mathrm{mV} / \mathrm{m}$, the lower-hybrid waves have peak amplitude $E_{\mathrm{LH}}=0.2 V_{0} B_{0} / c=10.8 \mathrm{mV} / \mathrm{m}$, while the ion cyclotron waves have peak amplitude $E_{\mathrm{ic}}=0.05$ $V_{0} B_{0} / c=2.7 \mathrm{mV} / \mathrm{m}$. Approximately half of the total plasma energy will be radiated in $4.7 \mathrm{sec}$. Assuming that $1.5 \mathrm{~kg}$ of barium are vaporized at $40 \%$ efficiency yields a total of $2.6 \times 10^{24}$ barium neutrals. With an ionization time of $29 \mathrm{sec}$, we have a total of $8.8 \times 10^{22}$ ions after $1 \mathrm{sec}$ and a total kinetic energy of $4.7 \times 10^{5} \mathrm{~J}$. Half of this total en- 
ergy is lost in $4.7 \mathrm{sec}$, yielding a total average radiated power of $50 \mathrm{~kW}$ across the entire frequency band. Note that we have chosen parameters that are typical of these experiments $1 \mathrm{sec}$ after the release. At these early times, the plasma density decreases at a rate proportional to $1 / t^{2}$ while the size of the cloud increases at a rate proportional to $t$. As a result, the slowing-down time is a constant. The fact, however, that momentum and energy are continually being added to the cloud by ionization of the neutral barium will alter our predictions somewhat. Detailed comparison of these results with observations is left to a future publication.

\section{SUMMARY}

The early-time ( $<100$ ion gyroperiods) evolution of plasmas moving across a background magnetic field has been addressed with a two-dimensional model in which a plasma cloud is assumed to have formed instantaneously with a velocity $V_{0}$ across a uniform background magnetic field and with a Gaussian density profile in the two dimensions perpendicular to the direction of motion. This model treats both the early-time dynamics associated with the formation of the polarization field and the generation and propagation of electromagnetic waves. A detailed description of the theory was presented in Paper I. In this paper, our analysis was applied to the modeling of low-beta, barium chemical releases in the magnetosphere.

In an effort to elucidate the essential role of the radiation field in this problem, we first examined in some detail the electrostatic limit of our analysis. In this limit, we were able to describe the development of the polarization field, the amplitude and frequency of its oscillations as a function of the local plasma density, and the partitioning of momentum and energy between the plasma and the polarization field also as a function of density. We found that, at high densities, the polarization field forms on an upper-hybrid time scale with a normalized amplitude $\left[E_{x} /\left(V_{0} B_{0} / c\right)\right]$ approximately equal to 1 , while at low densities the polarization field oscillates at both the electron and ion cyclotron frequencies but is dominated by ion cyclotron oscillations with a normalized amplitude that is much less than unity. An intermediate situation is achieved for densities between these two extremes, with oscillation frequencies ranging from the ion cyclotron frequency through the lower-hybrid range and up to the upper-hybrid frequency as density increases. The partitioning of momentum and energy is such that, in the high-density limit, the average momentum (energy) stored in the fields is small compared to the initial plasma momentum (energy) density and such that the fields store approximately the same relative amount of momentum and energy. In this regime, the ions and electrons undergo small oscillations about the initial direction of motion. In the low density limit, the plasma simply gyrates about an equilibrium position exchanging twice its initial momentum with the fields but very little of its energy. The amount of energy stored in the polarization field maximizes at densities such that the ion Debye length equals an ion gyroradius with an amplitude of $40 \%$ of the initial plasma kinetic energy.
During the early phases of evolution when the polarization field is developing, the electromagnetic results exhibit similar properties to those obtained in the electrostatic limit except that the oscillation frequencies of the fields are shifted slightly and two modes are present as a result of the generation of an induction field in the beam direction. The subsequent evolution of the plasma cloud, however, is dominated by radiation effects. At early times, high-frequency radiation emitted in the central portion of the plasma cloud carries away a small fraction of the plasma momentum and energy. As time progresses, the lower-frequency waves produced primarily in the outer layers result in the formation of several lower-hybrid whistler pulses that propagate through the interior of the plasma cloud and that lead ultimately to the slowing down of the plasma in these regions. A corresponding decrease in the "steady-state" polarization field occurs in the central portion of the cloud. At late times, times greater than an ion cyclotron period, the plasma relaxes into gyrotropic motion at frequencies somewhat less than the ion gyrofrequency. By this time, most of the plasma energy and momentum has been radiated away.

Scaling laws were presented and used to make quantitative predictions for low-beta magnetospheric barium releases. For the parameters given in Table $I$, a braking time of $\tau_{s}=4.2 \tau_{c i}$ or $4.7 \mathrm{sec}$ (where $\tau_{c i}=1.12 \mathrm{sec}$ ) was calculated. The peak amplitudes of fields emitted at high frequencies $\left(\omega>\omega_{c e}\right)$, at lower-hybrid frequencies $\left(\omega_{c i}<\omega<\omega_{c e}\right)$, and in the vicinity of the ion gyrofrequency were calculated to be approximately $54,10.8$, and 2.7 $\mathrm{mV} / \mathrm{m}$, respectively. It is predicted that approximately half of the total plasma energy will be radiated in $4.7 \mathrm{sec}$, yielding a total average radiated power of $50 \mathrm{~kW}$ across the entire frequency band.

The present analysis has direct application to the crossfield motion of plasmas in experiments where the background plasma density is small compared to the cloud density and where the cloud dynamic $\beta$ is small (e.g., magnetospheric chemical releases). The general application to active experiments and to space and astrophysical plasmas, however, will necessitate the inclusion of additional effects. First, the Hermite polynomial expansion will have to be taken to first order so that the $z$ electric field can be determined and so that improved accuracy can be obtained in modeling the outer layers of the plasma cloud. Second, the effects of a background plasma will have to be included. Third, plasma density and momentum production (as obtained, for example, in active experiments) will have to be modeled in order to treat the late time evolution of the plasma and electromagnetic radiation selfconsistently. Finally, expansion of the plasma dimensions with time (also obtained in active experiments) can also affect the self-consistent evolution of the plasma and radiation fields over long time scales. These issues will be addressed in future publications. 


\section{ACKNOWLEDGMENTS}

The authors would like to thank Dr. David J. Simons for many helpful discussions and for his support and encouragement throughout this effort.

Research at Los Alamos was performed under the auspices of the U.S. Department of Energy and was supported, in part, by the Office of Space Science and Applications (OSSA) of the National Aeronautics and Space Administration (NASA). This work was supported at the University of Michigan by NASA Grants No. NAGW2162 and No. NAGW-1619 and by the National Science Foundation (NSF) under Contract No. ATM-9114409.

${ }^{1}$ R. Roussel-Dupré and R. H. Miller, Phys. Fluids B 5, 1289 (1993).

${ }^{2}$ D. A. Gurnett, R. R. Anderson, T. Z. Ma, G. Haerendel,
G. Paschmann, O. H. Bauer, R. A. Treumann, H. C. Koons, R. H. Holzworth, and H. Luhr, J. Geophys. Res. 91, 10013 (1986).

${ }^{3}$ J. R. Wygant, P. S. Mozer, N. Maynard, H. Singer, S. B. Mende, W. Peterson, and R. Anderson, EOS Trans./Supplement 72, 407 (1991).

${ }^{4}$ M. C. Kelley, R. F. Pfaff, and G. Haerendel, J. Geophys. Res. 91, 9939 (1986).

${ }^{5}$ M. C. Kelley, C. M. Swenson, N. Brenning, K. Baker, and R. Pfaff, J. Geophys. Res. 96, 9703 (1991).

${ }^{6}$ C. M. Swenson, M. C. Kelley, F. Primdahl, and K. D. Baker, Geophys. Res. Lètt. 17, 2337 (1990).

${ }^{7}$ R. A. Treumann, L. Brostrom, J. LaBelle, and N. Sckopke, J. Geophys. Res. 95, 19099 (1990).

${ }^{8}$ P. A. Bernhardt, Phys. Fluids B 4, 2249 (1992).

${ }^{9}$ H. Dubner and J. Abate, J. Assoc. Comput. Mach. 15, 115 (1968).

${ }^{10}$ N. A. Krall and A. W. Trivelpiece, Principles of Plasma Physics (McGraw-Hill, New York, 1973).

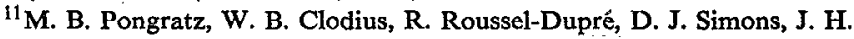
Wolcott, M. Baumback, P. Rodriguez, D. N. Walker, S. B. Mende, R. L. Rairden, H. C. Stenback-Nielsen, E. M. Wescott, and J. R. Wygant, EOS Trans./Supplement 72, 365 (1991). 